\title{
Encoding genetic circuits with DNA barcodes paves the way for machine learning-assisted metabolite biosensor response curve profiling in yeast
}

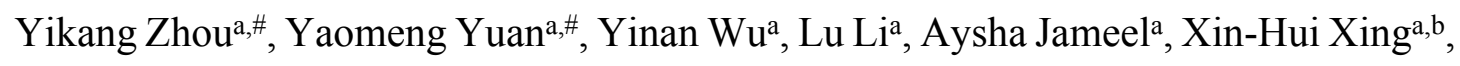
Chong Zhanga,b*

${ }^{a} \mathrm{MOE}$ Key Laboratory for Industrial Biocatalysis, Institute of Biochemical Engineering, Department of Chemical Engineering, Tsinghua University, Beijing 100084, China

${ }^{\mathrm{b} C e n t e r}$ for Synthetic and Systems Biology, Tsinghua University, Beijing 100084, China

\#These authors contributed equally to this work

*Address correspondence to Chong Zhang, chongzhang@tsinghua.edu.cn 
(a)

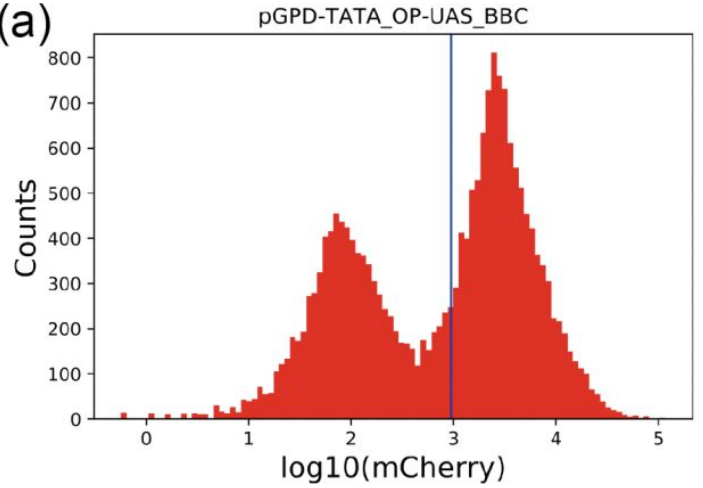

(b)

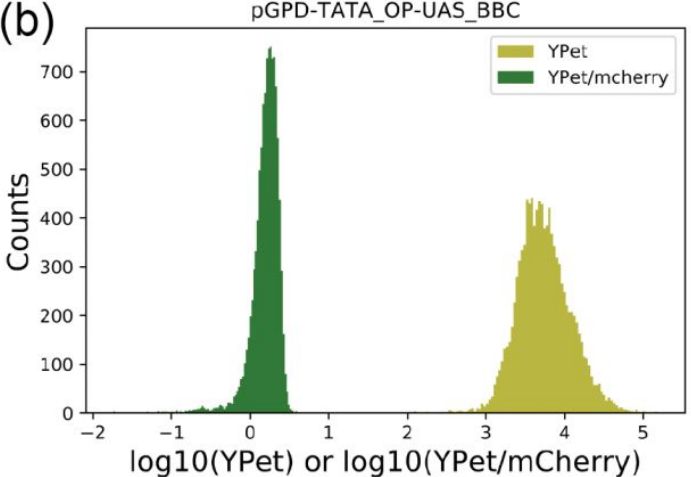

Figure S1 Cytometry analysis using pGPD-TATA_OP-UAS_BBC (SC-Ura). (a) The distribution of $\log _{10}$ (mCherry). The first peak corresponds to the cells losing plasmid, and the second peak corresponds to the cells with a single plasmid. (b) Comparison between response definition using $\log _{10}$ (YPet/mCherry) (used in the main paper, referred to as $\mu)$ and $\log _{10}($ YPet $)$. 
(a)

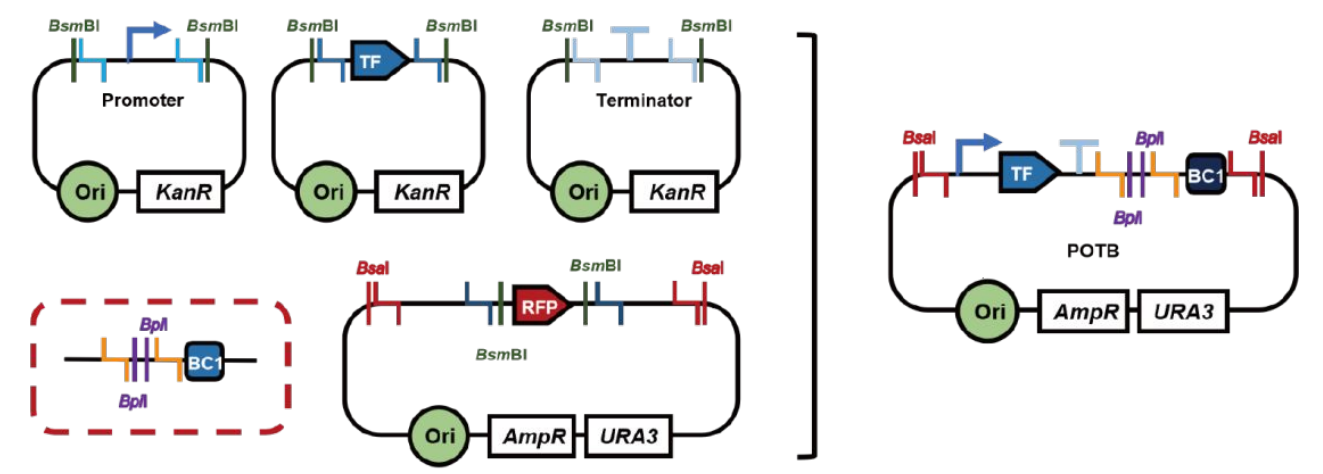

(b)

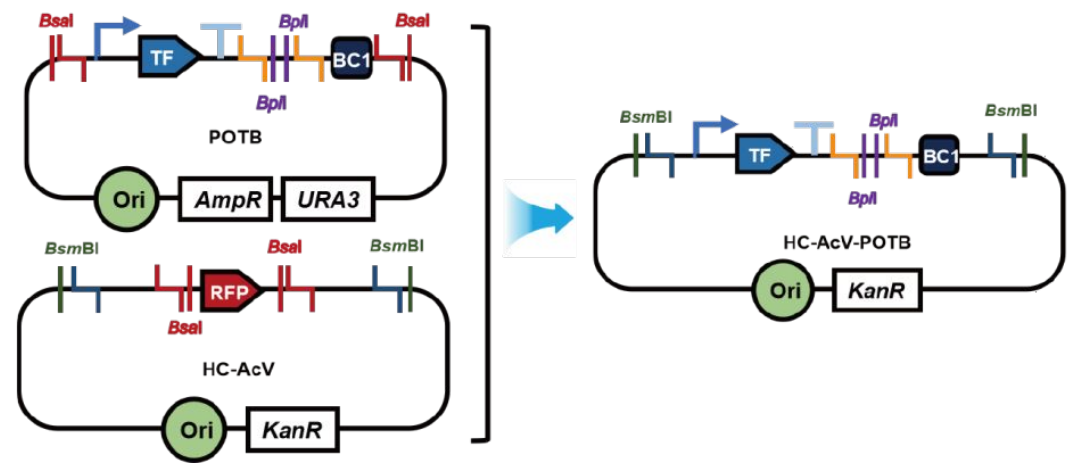

Figure S2 Trackable assembly-Step1. (a) The standardized promoter, transcription factor (TF), terminator, barcode linker and POT vector were assembled into the standard transcription unit PRO-ORF-TER-BC1 (POTB) by GoldenGate (BsmBI based) assembly. (b) The standard transcription unit POTB were transformed into the $\mathrm{HC}-\mathrm{AcV}$ vector to construct HC-AcV-POTB by GoldenGate (BsaI based) assembly. 


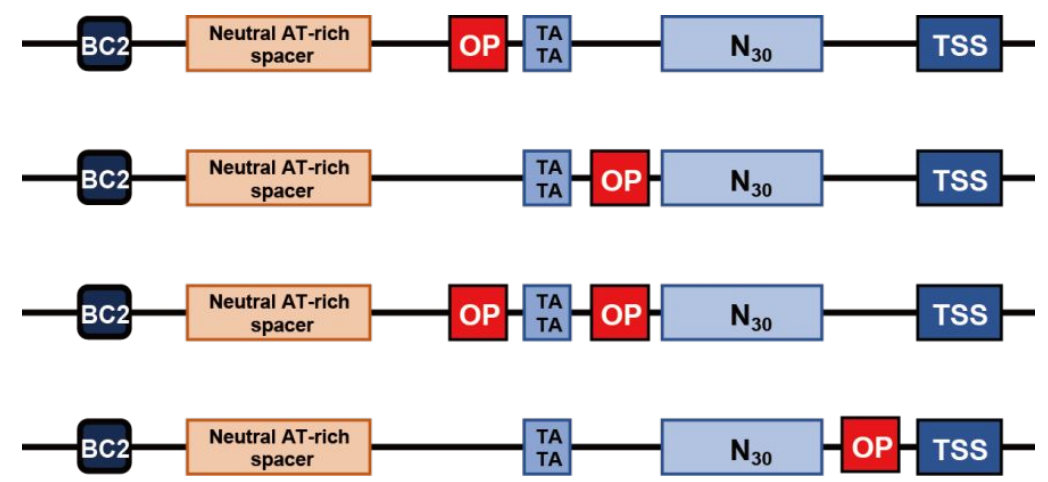

Figure S3 Trackable assembly-Step2. 4 different operators (OP) insertion schemes):

(1) directly downstream of the TATA-box (TATA_OP); (2) 1 bp before the TATAbox; (3) 1bp before and directly downstream of the TATA-box; (4) directly downstream of the N30 sequence (N30_OP). 
(a)

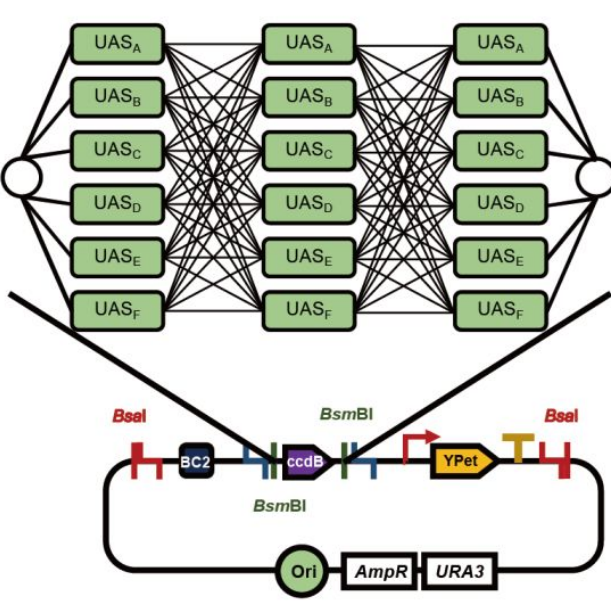

(b)

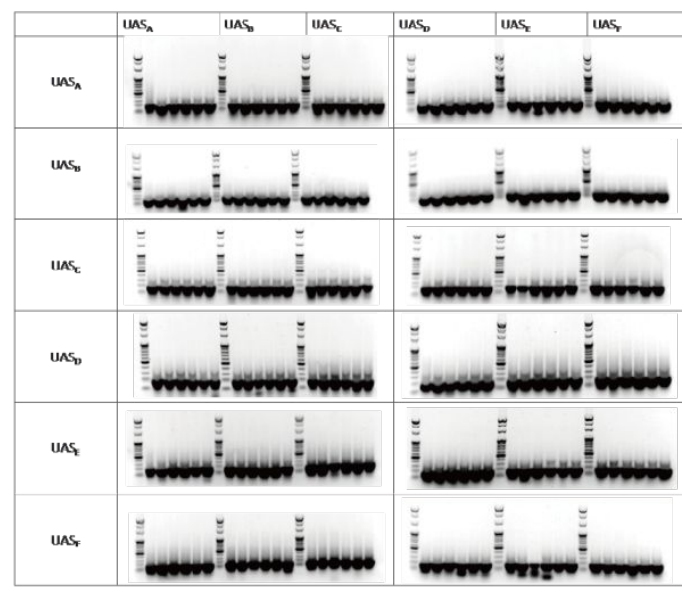

(c)

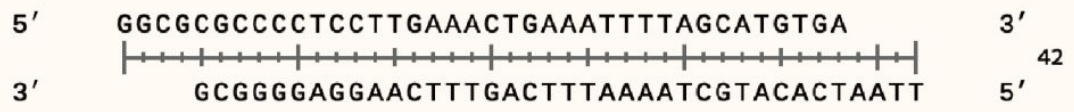

Figure S4 Trackable assembly-Step3. (a) Assemble the UAS sequence into the ccdBYPet-tPGK1 vector by GoldenGate (BsmBI) assembly; (b) Annealing of UAS linkers tested by agarose gel electrophoresis; (c) The UAS_FEC linker generated by annealing two DNA oligonucleotides. 
(a)

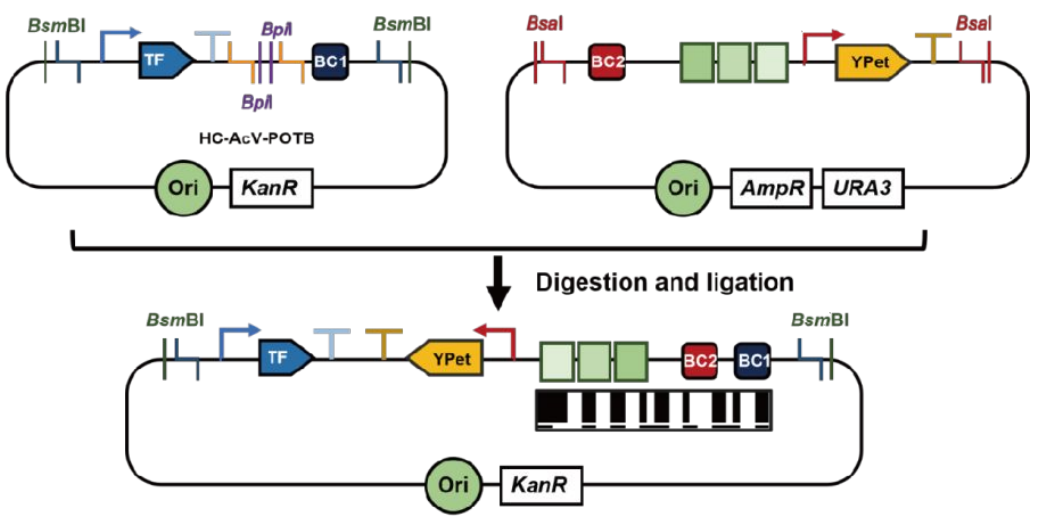

(b)

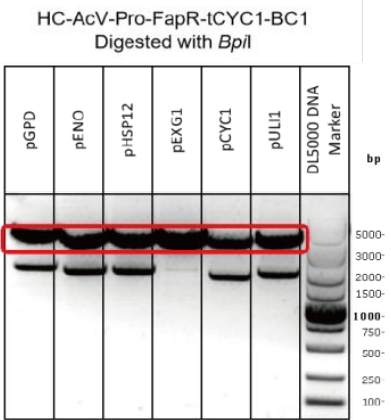

(c)

UAS-YPet-tPGK1

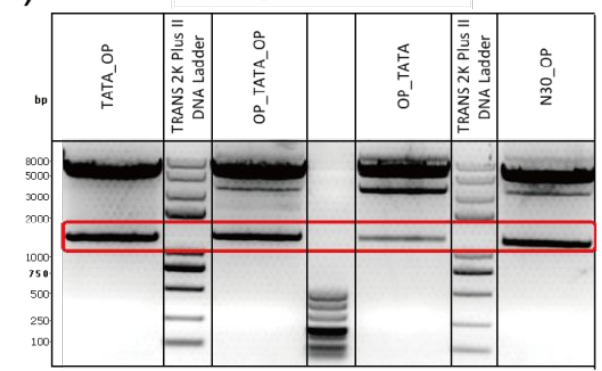

Figure S5 Trackable assembly-Step4. (a) Barcodes assembly: the UAS-YPet-tPGK1 fragment with BsaI digestion and HC-AcV-POTB vector with BpiI digestion were linked; (b) HC-AcV-POTB vector with BpiI digestion tested by agarose gel electrophoresis; (c) UAS-YPet-tPGK1 fragment with $B s a$ I digestion tested by agarose gel electrophoresis. Recycle the bands within the red frame box in (a), (b). 


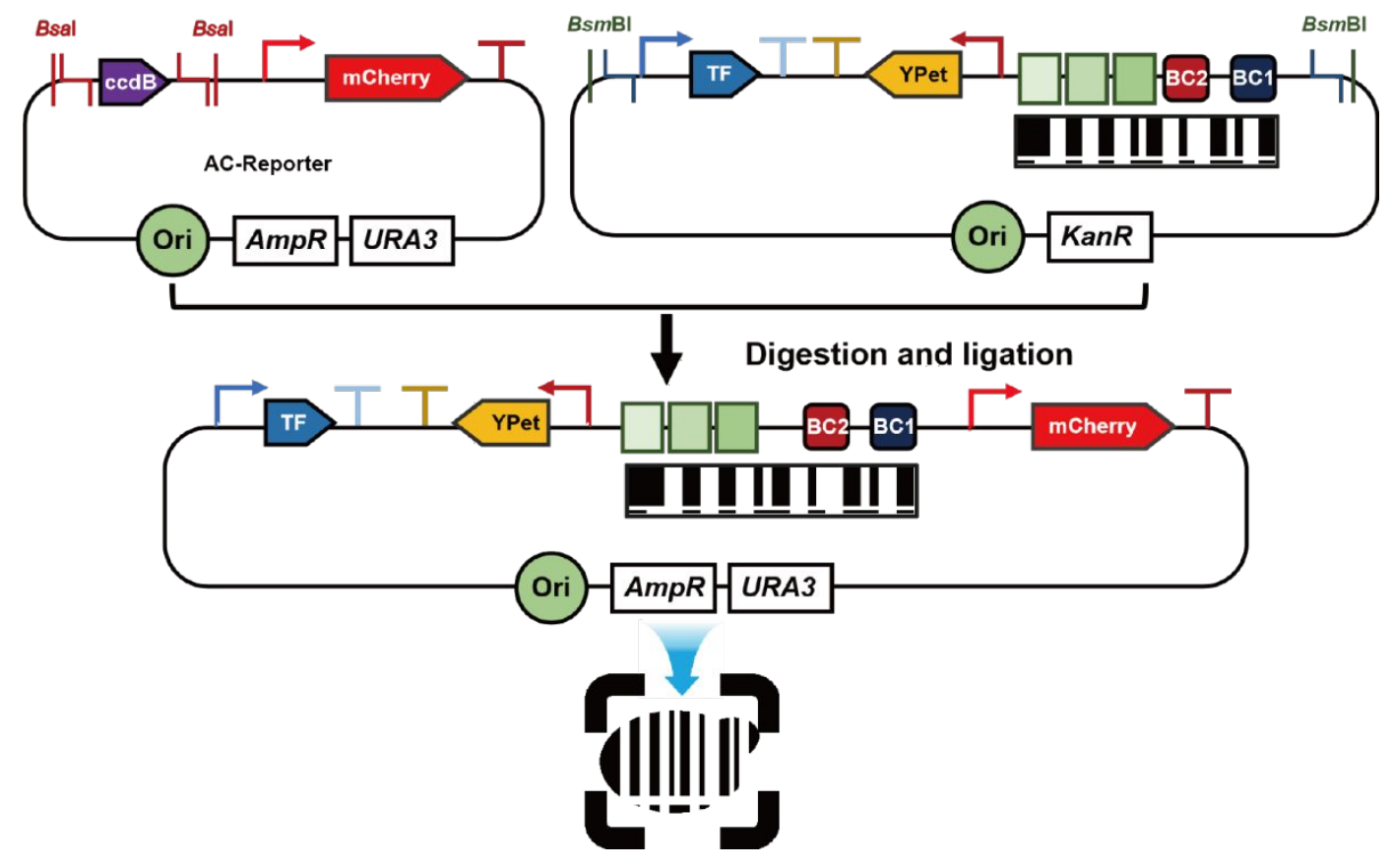

Figure S6 Linkage of HC-AcV-POT-BC1-BC2-UAS-YPet-tPGK1 fragment with Bsm BI digestion and AC-Reporter plasmid with $B s a \mathrm{I}$ digestion. The finally constructed plasmid library was transformed into BY4700. 
(a)

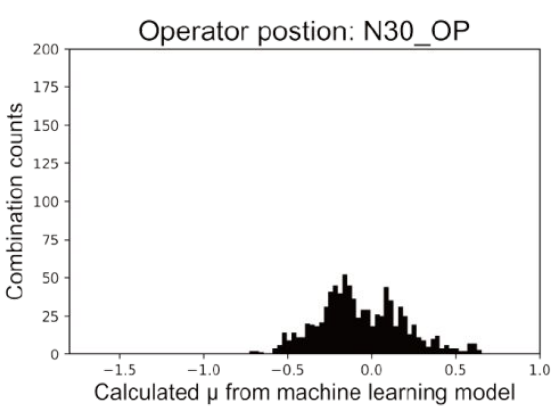

(c)

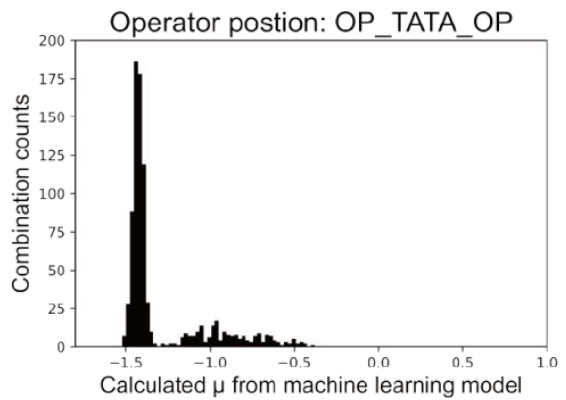

(b)

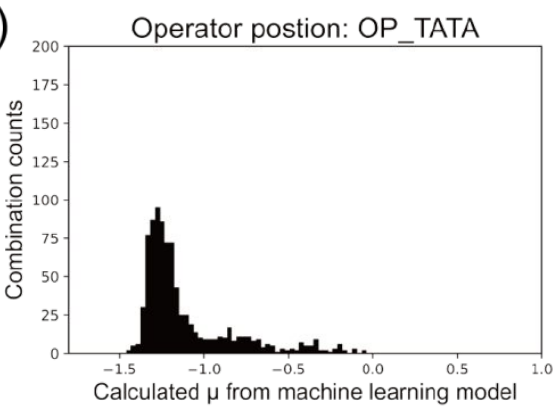

(d)

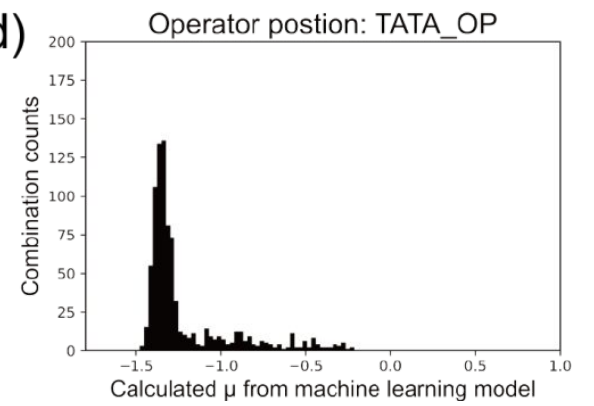

Figure S7 The predicted $\mu$ value distribution histograms of different operator

insertion schemes when no cerulenin was added. (a) N30_OP. (b) OP_TATA. (c) OP_TATA_OP. (d) TATA_OP. 
(a)
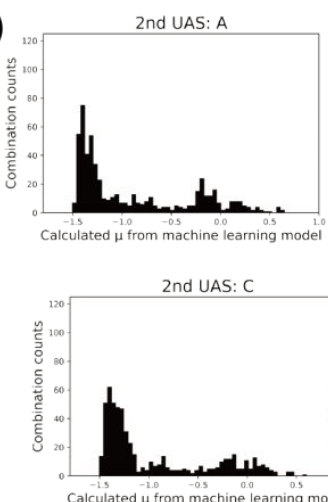

2nd UAS: E

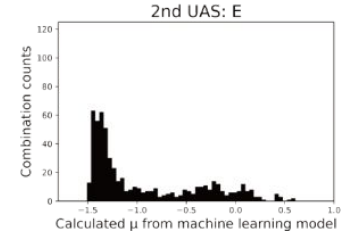

2nd UAS: B

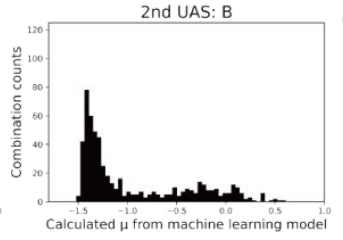

2nd UAS: D

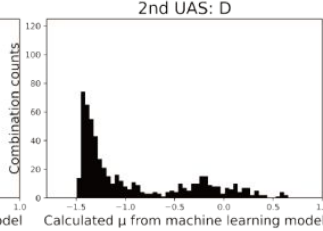

2nd UAS: F

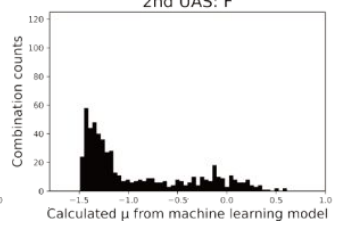

(b)

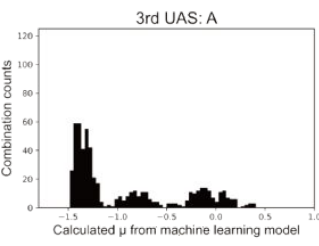

3rd UAS: C

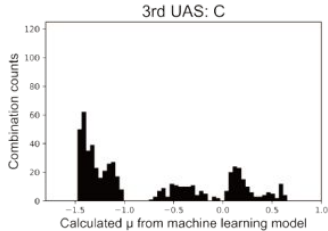

3rd UAS: E

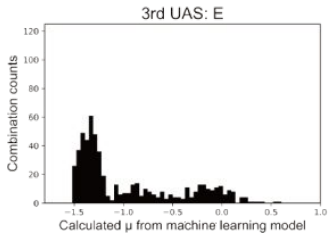

3rd UAS: B

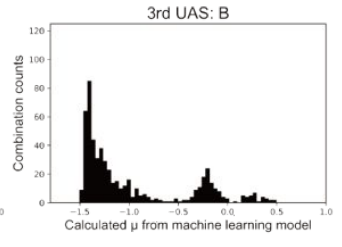

3rd UAS: D

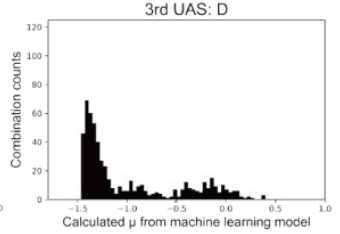

3rd UAS:F

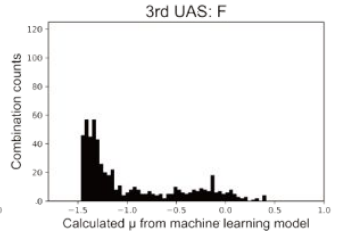

Figure S8 The predicted $\mu$ value distribution histograms of (a) 2nd UAS and (b) 3rd UAS when no cerulenin was added. 
(a)

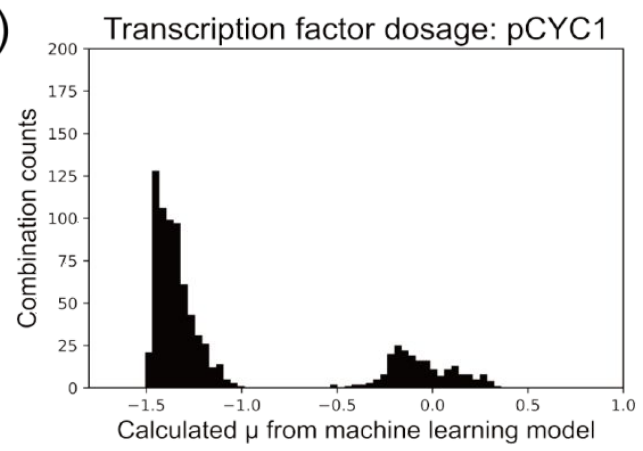

(c)

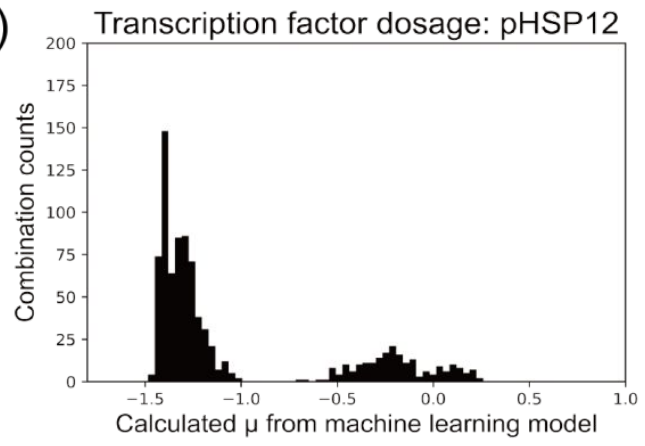

(b)

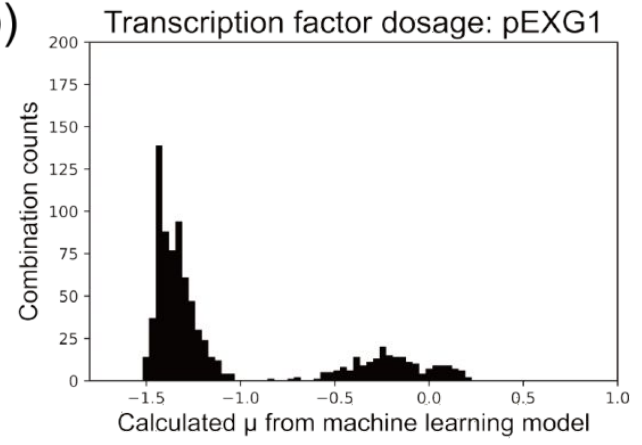

(d)

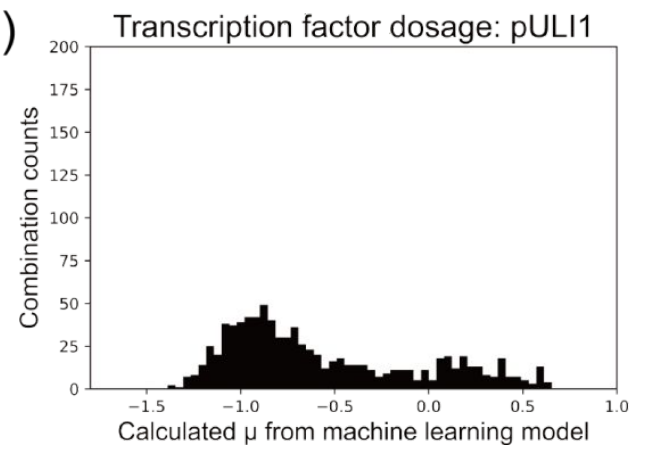

Figure S9 The predicted $\mu$ value distribution histograms of FapR expression driven by (a) $p H S P 12$, (b) $p E X G 1$, (c) $p C Y C 1$ and (d) $p U L I 1$ when no cerulenin was added. 
(a)

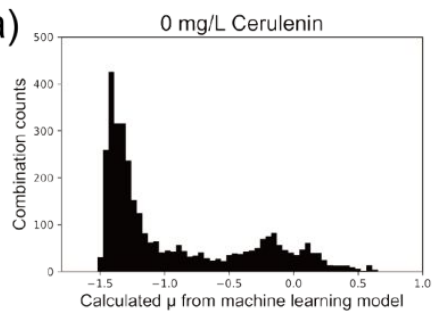

(d)

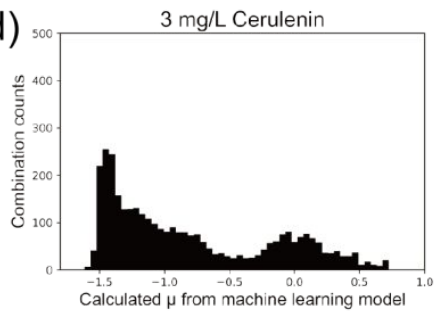

(b)

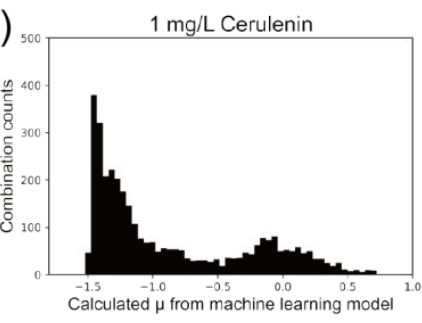

(e)

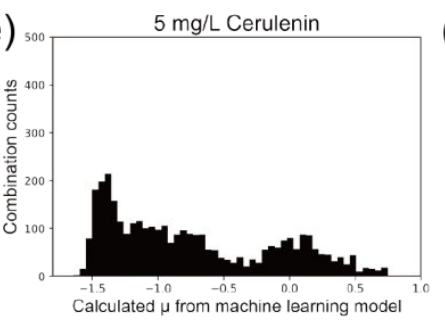

(c)
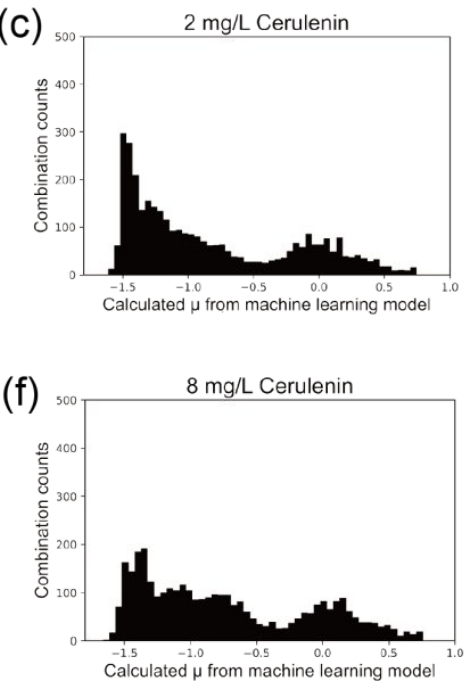

Figure S10 The predicted $\mu$ value distribution histograms in different cerulenin concentrations. 


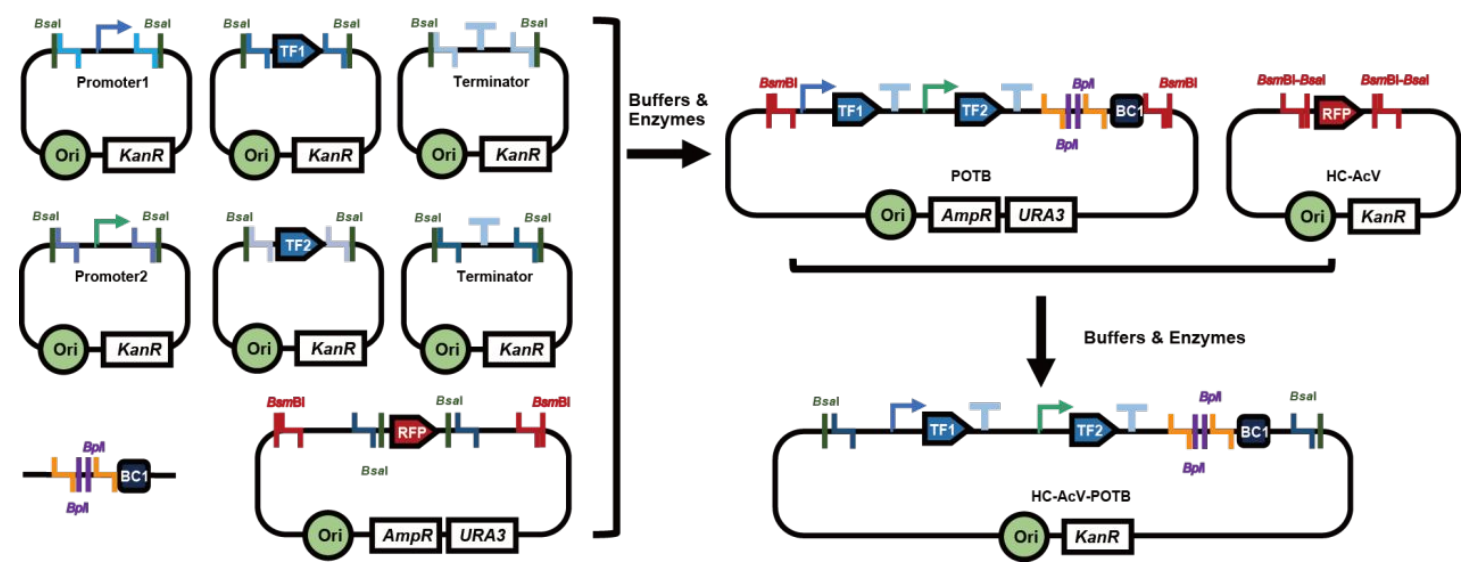

Figure S11 Modified Step 1 for the trackable assembly of AND logic biosensor, where an eight-fragment Goldengate is needed to assemble the sensing module with 2 TFs. Little change has to be made for the remaining steps. 


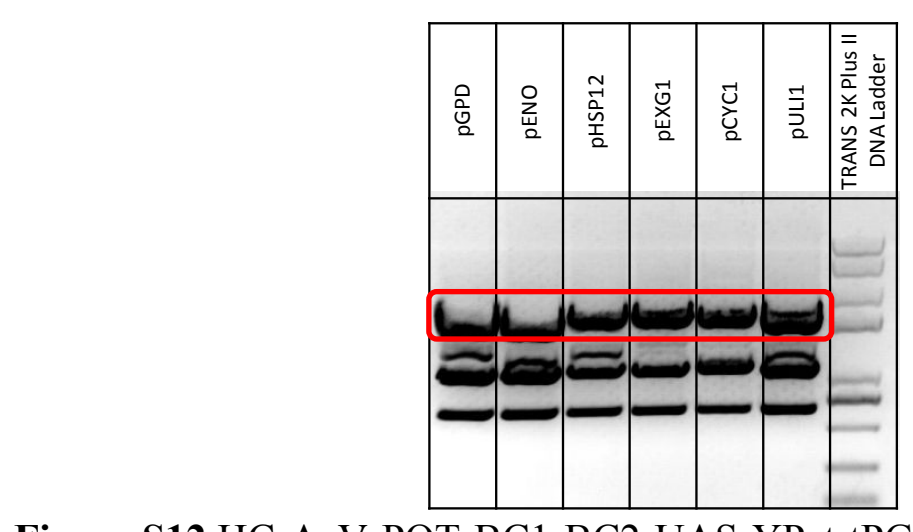

Figure S12 HC-AcV-POT-BC1-BC2-UAS-YPet-tPGK1 libraries with BsmBI

digestion tested by agarose gel electrophoresis 


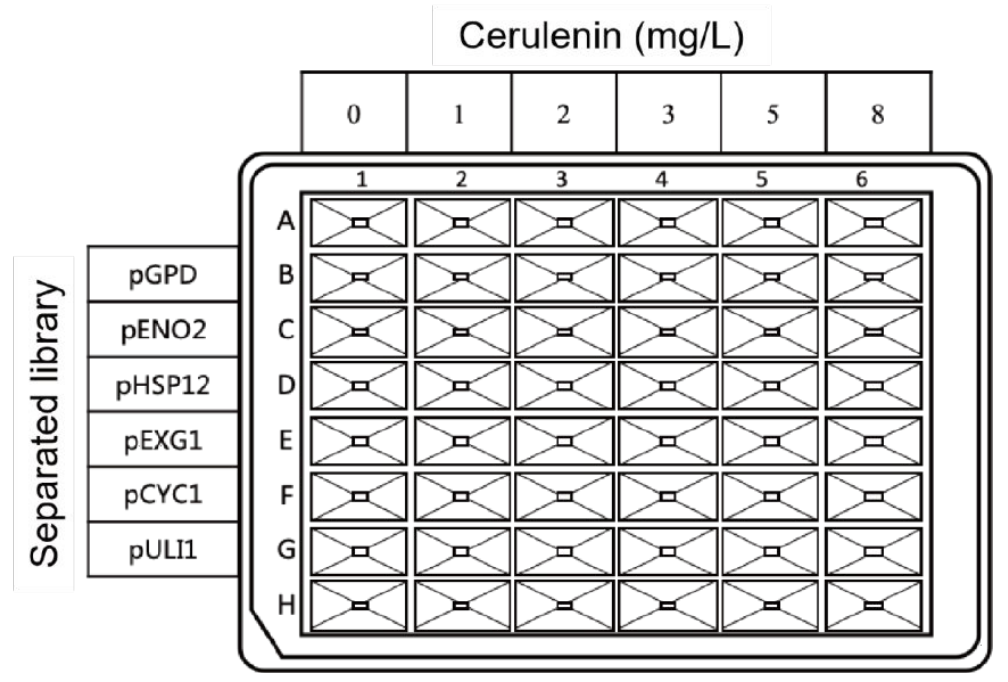

Figure S13 Schematic diagram of the distribution of FACS library samples in 48 deep well plates 
(a)

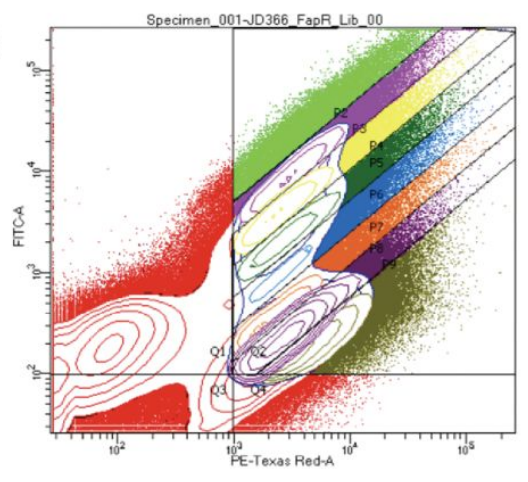

(b)

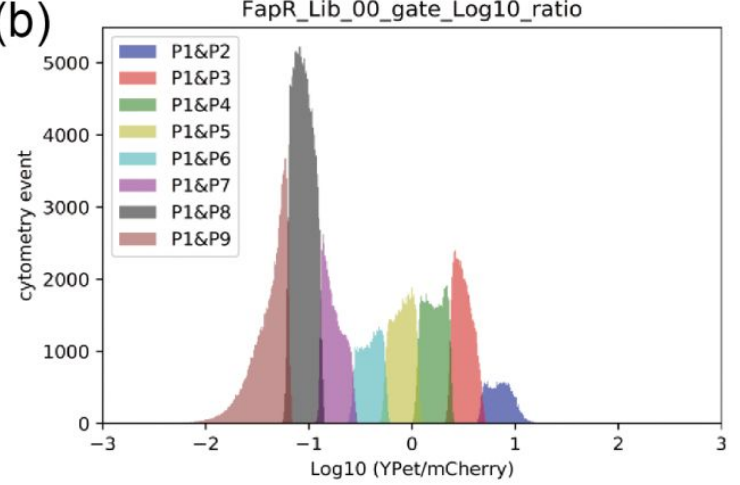

Figure S14 FACS sorting experiment of Lib_0A. (a) Gating of the cells into eight bins P2-P9 in sorting experiment. (b) The cell distribution of bins P2-P9 in $\log _{10}$ (YPet/mCherry) 
Table S1 The diversity analysis of the trackable assembly of TF-based metabolite biosensors in yeast

\begin{tabular}{|c|c|c|c|c|}
\hline & Name & Size & Transformants & Coverage \\
\hline Step 1 & $\begin{array}{c}\text { Sensing } \\
\text { module assembly }\end{array}$ & 6 & Individually & \\
\hline Step2 & $\begin{array}{l}\text { Reporter } \\
\text { module assembly- } \\
\text { operator insertion }\end{array}$ & 4 & Individually & \\
\hline Step3 & $\begin{array}{l}\text { Reporter } \\
\text { module assembly- } \\
\text { UAS assembly }\end{array}$ & 216 & $\sim 2 \times 10^{4}$ & $\sim 100$ \\
\hline Step4 & $\begin{array}{l}\text { Metabolite } \\
\text { biosensor } \\
\text { assembly-barcodes } \\
\text { assembly }\end{array}$ & 5184 & $\sim 2 \times 10^{5}$ & $\sim 40$ \\
\hline Step5 & $\begin{array}{l}\text { Transform } \\
\text { the genetic circuit } \\
\text { into yeast cell }\end{array}$ & 5184 & $\sim 2 \times 10^{5}$ & $\sim 40$ \\
\hline Finally & $\begin{array}{l}\text { Transform } \\
\text { the metablite } \\
\text { biosensors into } \\
\text { yeast cells }\end{array}$ & & Build 5155 (99.4\%) & \\
\hline
\end{tabular}


Table S2 The sequencing results of the colonies sampled from the mixed library of FapR-fapO based malony-CoA biosensor

\begin{tabular}{|c|c|c|c|c|c|c|}
\hline ID & Barcode1 & TF PRO & Barcode2 & $\begin{array}{c}\text { fap } O \text { insertion } \\
\text { scheme }\end{array}$ & UAS & True/False \\
\hline 1 & BC1_1 & $p U L I 1$ & $\mathrm{BC} 2 \_1$ & TATA_OP & $\mathrm{BFC}$ & False \\
\hline 2 & BC1_1 & $p C Y C 1$ & $\mathrm{BC} 2 \_1$ & TATA_OP & $\mathrm{BEC}$ & False \\
\hline 3 & $\mathrm{BC} 1 \_3$ & pHSP12 & $\mathrm{BC} 2 \_3$ & OP_TATA & $\mathrm{ABF}$ & True \\
\hline 4 & BC1_3 & pHSP12 & $\mathrm{BC} 2 \_1$ & TATA_OP & $\mathrm{BBF}$ & True \\
\hline 5 & BC1_3 & pHSP12 & $\mathrm{BC} 2 \_1$ & TATA_OP & $\mathrm{BBC}$ & True \\
\hline 6 & BC1_3 & pHSP12 & $\mathrm{BC} 2 \_1$ & TATA_OP & $* \mathrm{AD}$ & True \\
\hline 7 & $\mathrm{BC} 1 \_3$ & pHSP12 & $\mathrm{BC} 2 \_2$ & OP_TATA_OP & FAF & True \\
\hline 8 & BC1_3 & pHSP12 & $\mathrm{BC} 2 \_3$ & OP_TATA & CEC & True \\
\hline 9 & $\mathrm{BC} 1 \_3$ & pHSP12 & $\mathrm{BC} 2 \_3$ & OP_TATA & $\mathrm{CCC}$ & True \\
\hline 10 & $\mathrm{BC} 1 \_3$ & pHSP12 & $\mathrm{BC} 2 \_3$ & OP_TATA & $\mathrm{AFC}$ & True \\
\hline 11 & $\mathrm{BC} 1 \_3$ & pHSP12 & $\mathrm{BC} 2 \_3$ & OP_TATA_OP & $\mathrm{CDF}$ & True \\
\hline 12 & BC1_3 & $p E X G 1$ & BC2_4 & N30_OP & $\mathrm{EBE}$ & False \\
\hline 13 & BC1_3 & $p H S P 12$ & BC2_4 & N30_OP & $\mathrm{BBC}$ & True \\
\hline 14 & BC1_3 & pHSP12 & BC2_4 & N30_OP & EEC & True \\
\hline 15 & BC1_3 & $p H S P 12$ & BC2_4 & N30_OP & CAF & True \\
\hline 16 & BC1_4 & $p E X G 1$ & $\mathrm{BC} 2 \_1$ & TATA_OP & BAA & True \\
\hline 17 & BC1_4 & $p E X G 1$ & $\mathrm{BC} 2 \_1$ & TATA_OP & $\mathrm{CBF}$ & True \\
\hline 18 & BC1_4 & $p E X G 1$ & $\mathrm{BC} 2 \_1$ & TATA_OP & $\mathrm{ADC}$ & True \\
\hline 19 & BC1_4 & $p E X G 1$ & $\mathrm{BC} 2 \_1$ & TATA_OP & DEC & True \\
\hline 20 & BC1_4 & $p E X G 1$ & $\mathrm{BC} 2 \_2$ & OP_TATA_OP & $\mathrm{BBC}$ & True \\
\hline 21 & BC1_4 & $p E X G 1$ & $\mathrm{BC} 2 \_2$ & OP_TATA_OP & $\mathrm{CBC}$ & True \\
\hline 22 & BC1_4 & $p U L I 1$ & $\mathrm{BC} 2 \_2$ & OP_TATA_OP & DFF & False \\
\hline 23 & BC1_4 & $p E X G 1$ & $\mathrm{BC} 2 \_3$ & OP_TATA & $\mathrm{AFC}$ & True \\
\hline 24 & BC1_4 & $p E X G 1$ & $\mathrm{BC} 2 \_3$ & OP_TATA & EBC & True \\
\hline 25 & BC1_4 & $p E X G 1$ & $\mathrm{BC} 2 \_3$ & OP_TATA & EAD & True \\
\hline 26 & BC1_4 & $p E X G 1$ & $\mathrm{BC} 2 \_3$ & OP_TATA & $\mathrm{ADF}$ & True \\
\hline 27 & BC1_4 & $p C Y C 1$ & BC2_4 & N30_OP & DEC & False \\
\hline 28 & BC1_5 & $p C Y C 1$ & $\mathrm{BC} 2 \_1$ & TATA_OP & $\mathrm{FCF}$ & True \\
\hline 29 & BC1_5 & $p C Y C 1$ & BC2_1 & TATA_OP & FBF & True \\
\hline 30 & BC1_5 & $p C Y C 1$ & $\mathrm{BC} 2 \_1$ & TATA_OP & EEF & True \\
\hline
\end{tabular}




\begin{tabular}{|c|c|c|c|c|c|c|}
\hline ID & Barcode1 & TF PRO & Barcode2 & $\begin{array}{c}\text { fapO insertion } \\
\text { scheme }\end{array}$ & UAS & True/False \\
\hline 31 & $\mathrm{BC} 1 \_5$ & $p C Y C 1$ & BC2_1 & TATA_OP & DCD & True \\
\hline 32 & $\mathrm{BC} 1 \_5$ & $p C Y C 1$ & BC2_1 & TATA_OP & DAC & True \\
\hline 33 & $\mathrm{BC} 1 \_5$ & $p C Y C 1$ & BC2_1 & TATA_OP & DAC & True \\
\hline 34 & BC1_5 & $p C Y C 1$ & BC2_1 & TATA_OP & $\mathrm{BDC}$ & True \\
\hline 35 & $\mathrm{BC} 1 \_5$ & $p C Y C 1$ & BC2_1 & TATA_OP & $\mathrm{BAC}$ & True \\
\hline 36 & $\mathrm{BC} 1 \_5$ & $p C Y C 1$ & BC2_1 & TATA_OP & CAC & True \\
\hline 37 & $\mathrm{BC} 1 \_5$ & $p C Y C 1$ & BC2_1 & TATA_OP & FAC & True \\
\hline 38 & BC1_5 & $p C Y C 1$ & BC2_1 & TATA_OP & AFF & True \\
\hline 39 & $\mathrm{BC} 1 \_5$ & $p C Y C 1$ & BC2_1 & TATA_OP & $\mathrm{ABC}$ & True \\
\hline 40 & $\mathrm{BC} 1 \_5$ & $p C Y C 1$ & BC2_1 & TATA_OP & $\mathrm{BCC}$ & True \\
\hline 41 & $\mathrm{BC} 1 \_5$ & $p C Y C 1$ & BC2_1 & TATA_OP & FEC & True \\
\hline 42 & BC1_5 & $p C Y C 1$ & BC2_1 & TATA_OP & AFD & True \\
\hline 43 & $\mathrm{BC} 1 \_5$ & $p C Y C 1$ & BC2_1 & TATA_OP & $\mathrm{BAC}$ & True \\
\hline 44 & $\mathrm{BC} 1 \_5$ & $p C Y C 1$ & BC2_1 & TATA_OP & FAF & True \\
\hline 45 & BC1_5 & $p C Y C 1$ & BC2_2 & OP_TATA_OP & DBC & True \\
\hline 46 & BC1_5 & $p C Y C 1$ & BC2_2 & OP_TATA_OP & $\mathrm{CFC}$ & True \\
\hline 47 & $\mathrm{BC} 1 \_5$ & $p C Y C 1$ & BC2_2 & OP_TATA_OP & $\mathrm{BFB}$ & True \\
\hline 48 & $\mathrm{BC} 1 \_5$ & $p C Y C 1$ & BC2_2 & OP_TATA_OP & $\mathrm{CCC}$ & True \\
\hline 49 & $\mathrm{BC} 1 \_5$ & $p C Y C 1$ & BC2_2 & OP_TATA_OP & DBF & True \\
\hline 50 & $\mathrm{BC} 1 \_5$ & $p C Y C 1$ & BC2_2 & OP_TATA_OP & $\mathrm{ACC}$ & True \\
\hline 51 & $\mathrm{BC} 1 \_5$ & $p C Y C 1$ & BC2_2 & OP_TATA_OP & $\mathrm{ABC}$ & True \\
\hline 52 & $\mathrm{BC} 1 \_5$ & $p C Y C 1$ & BC2_2 & OP_TATA_OP & BAF & True \\
\hline 53 & $\mathrm{BC} 1 \_5$ & $p C Y C 1$ & BC2_2 & OP_TATA_OP & DFC & True \\
\hline 54 & $\mathrm{BC} 1 \_5$ & $p C Y C 1$ & BC2_2 & OP_TATA_OP & $\mathrm{BBD}$ & True \\
\hline 55 & $\mathrm{BC} 1 \_5$ & $p C Y C 1$ & BC2_2 & OP_TATA_OP & $\mathrm{ADC}$ & True \\
\hline 56 & $\mathrm{BC} 1 \_5$ & $p C Y C 1$ & BC2_2 & OP_TATA_OP & FBC & True \\
\hline 57 & $\mathrm{BC} 1 \_5$ & $p C Y C 1$ & $\mathrm{BC} 2 \_3$ & OP_TATA & $\mathrm{CBC}$ & True \\
\hline 58 & $\mathrm{BC} 1 \_5$ & $p C Y C 1$ & $\mathrm{BC} 2 \_3$ & OP_TATA & $\mathrm{AEF}$ & True \\
\hline 59 & $\mathrm{BC} 1 \_5$ & $p C Y C 1$ & $\mathrm{BC} 2 \_3$ & OP_TATA & EDF & True \\
\hline 60 & BC1_5 & $p C Y C 1$ & $\mathrm{BC} 2 \_3$ & OP_TATA & $\mathrm{BEC}$ & True \\
\hline 61 & $\mathrm{BC} 1 \_5$ & $p C Y C 1$ & $\mathrm{BC} 2 \_3$ & OP_TATA & $\mathrm{ABC}$ & True \\
\hline 62 & $\mathrm{BC} 1 \_5$ & $p C Y C 1$ & $\mathrm{BC} 2 \_3$ & OP_TATA & EAD & True \\
\hline 63 & $\mathrm{BC} 1 \_5$ & $p C Y C 1$ & $\mathrm{BC} 2 \_3$ & OP_TATA & $\mathrm{CDC}$ & True \\
\hline
\end{tabular}




\begin{tabular}{|c|c|c|c|c|c|c|}
\hline ID & Barcode1 & TF PRO & Barcode2 & $\begin{array}{c}\text { fapO insertion } \\
\text { scheme }\end{array}$ & UAS & True/False \\
\hline 64 & BC1_5 & $p C Y C 1$ & $\mathrm{BC} 2 \_3$ & OP_TATA & DEC & True \\
\hline 65 & BC1_5 & $p E X G 1$ & $\mathrm{BC} 2 \_3$ & OP_TATA & $\mathrm{BDC}$ & True \\
\hline 66 & BC1_5 & $p C Y C 1$ & $\mathrm{BC} 2 \_3$ & OP_TATA & DDC & True \\
\hline 67 & BC1_5 & $p C Y C 1$ & $\mathrm{BC} 2 \_3$ & OP_TATA & $\mathrm{ADC}$ & True \\
\hline 68 & $\mathrm{BC} 1 \_5$ & $p C Y C 1$ & $\mathrm{BC} 2 \_4$ & N30_OP & $\mathrm{CDF}$ & True \\
\hline 69 & BC1_5 & $p C Y C 1$ & $\mathrm{BC} 2 \_4$ & N30_OP & CAE & True \\
\hline 70 & BC1_5 & $p C Y C 1$ & $\mathrm{BC} 2 \_4$ & N30_OP & FDC & True \\
\hline 71 & BC1_5 & $p C Y C 1$ & $\mathrm{BC} 2 \_4$ & N30_OP & BAF & True \\
\hline 72 & BC1_5 & $p C Y C 1$ & $\mathrm{BC} 2 \_4$ & N30_OP & CAC & True \\
\hline 73 & BC1_5 & $p C Y C 1$ & $\mathrm{BC} 2 \_4$ & N30_OP & DCD & True \\
\hline 74 & BC1_5 & $p C Y C 1$ & $\mathrm{BC} 2 \_4$ & N30_OP & $\mathrm{ADC}$ & True \\
\hline 75 & BC1_5 & $p C Y C 1$ & $\mathrm{BC} 2 \_4$ & N30_OP & EBC & True \\
\hline 76 & BC1_5 & $p C Y C 1$ & $\mathrm{BC} 2 \_4$ & N30_OP & CAC & True \\
\hline 77 & BC1_5 & $p C Y C 1$ & $\mathrm{BC} 2 \_4$ & N30_OP & $\mathrm{F}^{*} \mathrm{~F}$ & True \\
\hline 78 & BC1_5 & $p C Y C 1$ & $\mathrm{BC} 2 \_4$ & N30_OP & FCC & True \\
\hline 79 & BC1_5 & $p C Y C 1$ & $\mathrm{BC} 2 \_4$ & N30_OP & $\mathrm{ABD}$ & True \\
\hline 80 & BC1_5 & $p C Y C 1$ & $\mathrm{BC} 2 \_4$ & N30_OP & $\mathrm{ABF}$ & True \\
\hline 81 & BC1_5 & $p C Y C 1$ & $\mathrm{BC} 2 \_4$ & N30_OP & $\mathrm{BDC}$ & True \\
\hline 82 & BC1_6 & $p U L I 1$ & $\mathrm{BC} 2 \_1$ & TATA_OP & $\mathrm{BFC}$ & True \\
\hline 83 & BC1_6 & $p U L I 1$ & $\mathrm{BC} 2 \_1$ & TATA_OP & $\mathrm{CBC}$ & True \\
\hline 84 & BC1_6 & $p U L I 1$ & BC2_1 & TATA_OP & DDF & True \\
\hline 85 & $\mathrm{BC} 1 \_6$ & $p U L I 1$ & $\mathrm{BC} 2 \_1$ & TATA_OP & CAC & True \\
\hline 86 & BC1_6 & $p U L I 1$ & $\mathrm{BC} 2 \_1$ & TATA_OP & $\mathrm{BDA}$ & True \\
\hline 87 & BC1_6 & $p U L I 1$ & BC2_1 & TATA_OP & AED & True \\
\hline 88 & BC1_6 & $p U L I 1$ & BC2_1 & TATA_OP & $\mathrm{EBF}$ & True \\
\hline 89 & BC1_6 & pULII & $\mathrm{BC} 2 \_1$ & TATA_OP & $\mathrm{CBC}$ & True \\
\hline 90 & BC1_6 & $p U L I 1$ & $\mathrm{BC} 2 \_1$ & TATA_OP & CAC & True \\
\hline 91 & BC1_6 & $p U L I 1$ & BC2_1 & TATA_OP & DBE & True \\
\hline 92 & BC1_6 & $p U L I 1$ & $\mathrm{BC} 2 \_1$ & TATA_OP & $\mathrm{CBC}$ & True \\
\hline 93 & BC1_6 & pULII & $\mathrm{BC} 2 \_1$ & TATA_OP & FAD & True \\
\hline 94 & BC1_6 & $p U L I 1$ & BC2_1 & TATA_OP & $\mathrm{ABC}$ & True \\
\hline 95 & BC1_6 & $p U L I 1$ & BC2_1 & TATA_OP & $\mathrm{FBC}$ & True \\
\hline 96 & BC1_6 & $p U L I 1$ & BC2_1 & TATA_OP & CAC & True \\
\hline
\end{tabular}




\begin{tabular}{|c|c|c|c|c|c|c|}
\hline ID & Barcode1 & TF PRO & Barcode2 & $\begin{array}{l}\text { fapO insertion } \\
\text { scheme }\end{array}$ & UAS & True/False \\
\hline 97 & BC1_6 & $p U L I 1$ & $\mathrm{BC} 2 \_1$ & TATA_OP & FED & True \\
\hline 98 & BC1_6 & $p U L I 1$ & $\mathrm{BC} 2 \_2$ & OP_TATA_OP & $\mathrm{CEF}$ & True \\
\hline 99 & $\mathrm{BC} 1 \_6$ & $p U L I 1$ & $\mathrm{BC} 2 \_2$ & OP_TATA_OP & $\mathrm{CDC}$ & True \\
\hline 100 & BC1_6 & $p U L I 1$ & $\mathrm{BC} 2 \_2$ & OP_TATA_OP & CDD & True \\
\hline 101 & BC1_6 & $p U L I I$ & $\mathrm{BC} 2 \_2$ & OP_TATA_OP & $\mathrm{BEF}$ & True \\
\hline 102 & $\mathrm{BC} 1 \_6$ & pULII & $\mathrm{BC} 2 \_2$ & OP_TATA_OP & $\mathrm{CCD}$ & True \\
\hline 103 & BC1_6 & $p U L I 1$ & $\mathrm{BC} 2 \_2$ & OP_TATA_OP & BDD & True \\
\hline 104 & BC1_6 & $p C Y C 1$ & $\mathrm{BC} 2 \_2$ & OP_TATA_OP & DDE & False \\
\hline 105 & BC1_6 & $p U L I 1$ & $\mathrm{BC} 2 \_2$ & OP_TATA_OP & EBC & True \\
\hline 106 & BC1_6 & pULII & $\mathrm{BC} 2 \_2$ & OP_TATA_OP & $\mathrm{BAC}$ & True \\
\hline 107 & BC1_6 & $p U L I 1$ & $\mathrm{BC} 2 \_2$ & OP_TATA_OP & BAF & True \\
\hline 108 & BC1_6 & $p U L I 1$ & BC2_2 & OP_TATA_OP & $\mathrm{FBC}$ & True \\
\hline 109 & BC1_6 & $p U L I 1$ & $\mathrm{BC} 2 \_3$ & OP_TATA & $\mathrm{F}^{*} \mathrm{~F}$ & True \\
\hline 110 & BC1_6 & $p U L I 1$ & $\mathrm{BC} 2 \_3$ & OP_TATA & FEC & True \\
\hline 111 & BC1_6 & $p U L I 1$ & $\mathrm{BC} 2 \_3$ & OP_TATA & $\mathrm{BAC}$ & True \\
\hline 112 & BC1_6 & $p U L I 1$ & $\mathrm{BC} 2 \_3$ & OP_TATA & $\mathrm{BDC}$ & True \\
\hline 113 & BC1_6 & $p U L I 1$ & $\mathrm{BC} 2 \_3$ & OP_TATA & $\mathrm{AEF}$ & True \\
\hline 114 & BC1_6 & $p U L I 1$ & $\mathrm{BC} 2 \_3$ & OP_TATA & CAC & True \\
\hline 115 & $\mathrm{BC} 1 \_6$ & $p U L I 1$ & $\mathrm{BC} 2 \_3$ & OP_TATA & DAF & True \\
\hline 116 & BC1_6 & $p U L I 1$ & $\mathrm{BC} 2 \_3$ & OP_TATA & $\mathrm{EBC}$ & True \\
\hline 117 & BC1_6 & $p U L I I$ & $\mathrm{BC} 2 \_3$ & OP_TATA & BED & True \\
\hline 118 & BC1_6 & $p U L I 1$ & $\mathrm{BC} 2 \_3$ & OP_TATA & AAF & True \\
\hline 119 & BC1_6 & $p U L I 1$ & $\mathrm{BC} 2 \_3$ & OP_TATA & EDC & True \\
\hline 120 & BC1_6 & pULII & $\mathrm{BC} 2 \_4$ & N30_OP & $\mathrm{ABF}$ & True \\
\hline 121 & BC1_6 & $p U L I 1$ & $\mathrm{BC} 2 \_4$ & N30_OP & $\mathrm{BBD}$ & True \\
\hline 122 & BC1_6 & $p U L I 1$ & $\mathrm{BC} 2 \_4$ & N30_OP & BDC & True \\
\hline 123 & BC1_6 & $p U L I 1$ & $\mathrm{BC} 2 \_4$ & N30_OP & $\mathrm{AAC}$ & True \\
\hline 124 & BC1_6 & pULII & $\mathrm{BC} 2 \_4$ & N30_OP & EAF & True \\
\hline 125 & BC1_6 & $p U L I 1$ & $\mathrm{BC} 2 \_4$ & N30_OP & BFD & True \\
\hline 126 & BC1_6 & $p U L I 1$ & $\mathrm{BC} 2 \_4$ & N30_OP & BFD & True \\
\hline 127 & BC1_6 & $p U L I 1$ & $\mathrm{BC} 2 \_4$ & N30_OP & $* A C$ & True \\
\hline 128 & BC1_6 & $p U L I 1$ & $\mathrm{BC} 2 \_4$ & N30_OP & $\mathrm{BCA}$ & True \\
\hline
\end{tabular}


Table S3 The sequencing results of the colonies sampled from the separated library for FapR-fapO based malony-CoA biosensor

\begin{tabular}{|c|c|c|c|c|c|c|}
\hline ID & Barcode1 & TF PRO & Barcode2 & fapO insertion scheme & UAS & True/False \\
\hline 1 & BC1_1 & $p G P D$ & $\mathrm{BC} 2 \_3$ & OP_TATA & $\mathrm{FBC}$ & True \\
\hline 2 & BC1_1 & $p G P D$ & $\mathrm{BC} 2 \_3$ & OP_TATA & FBC & True \\
\hline 3 & BC1_1 & $p G P D$ & $\mathrm{BC} 2 \_3$ & OP_TATA & FBC & True \\
\hline 4 & BC1_1 & $p G P D$ & $\mathrm{BC} 2 \_3$ & OP_TATA & FBC & True \\
\hline 5 & BC1_1 & $p G P D$ & $\mathrm{BC} 2 \_3$ & OP_TATA & FBC & True \\
\hline 6 & BC1_2 & $p E N O 2$ & $\mathrm{BC} 2 \_1$ & TATA_OP & $\mathrm{ECC}$ & True \\
\hline 7 & BC1_2 & $p E N O 2$ & $\mathrm{BC} 2 \_3$ & OP_TATA & $\mathrm{BEC}$ & True \\
\hline 8 & BC1_2 & $p E N O 2$ & $\mathrm{BC} 2 \_3$ & OP_TATA & $\mathrm{BEF}$ & True \\
\hline 9 & $\mathrm{BC} 1 \_3$ & pHSP12 & $\mathrm{BC} 2 \_1$ & TATA_OP & $\mathrm{BBC}$ & True \\
\hline 10 & BC1_3 & pHSP12 & $\mathrm{BC} 2 \_1$ & TATA_OP & $\mathrm{BBC}$ & True \\
\hline 11 & BC1_3 & pHSP12 & $\mathrm{BC} 2 \_1$ & TATA_OP & $\mathrm{ABD}$ & True \\
\hline 12 & BC1_3 & pHSP12 & $\mathrm{BC} 2 \_1$ & TATA_OP & $\mathrm{BDC}$ & True \\
\hline 13 & BC1_3 & pHSP12 & $\mathrm{BC} 2 \_1$ & TATA_OP & $\mathrm{BDC}$ & True \\
\hline 14 & BC1_3 & pHSP12 & $\mathrm{BC} 2 \_1$ & TATA_OP & FAF & True \\
\hline 15 & BC1_3 & pHSP12 & $\mathrm{BC} 2 \_1$ & TATA_OP & FAF & True \\
\hline 16 & BC1_3 & pHSP12 & BC2_1 & TATA_OP & $\mathrm{ABD}$ & True \\
\hline 17 & BC1_3 & pHSP12 & BC2_1 & TATA_OP & DEC & True \\
\hline 18 & BC1_3 & pHSP12 & $\mathrm{BC} 2 \_1$ & TATA_OP & $\mathrm{EBF}$ & True \\
\hline 19 & BC1_3 & pHSP12 & $\mathrm{BC} 2 \_1$ & TATA_OP & $\mathrm{AAC}$ & True \\
\hline 20 & BC1_3 & pHSP12 & $\mathrm{BC} 2 \_1$ & TATA_OP & $\mathrm{BDC}$ & True \\
\hline 21 & BC1_3 & pHSP12 & $\mathrm{BC} 2 \_1$ & TATA_OP & BAF & True \\
\hline 22 & BC1_3 & pHSP12 & $\mathrm{BC} 2 \_1$ & TATA_OP & $\mathrm{FBC}$ & True \\
\hline 23 & $\mathrm{BC} 1 \_3$ & pHSP12 & $\mathrm{BC} 2 \_1$ & TATA_OP & $\mathrm{ABF}$ & True \\
\hline 24 & BC1_3 & pHSP12 & $\mathrm{BC} 2 \_1$ & TATA_OP & DFF & True \\
\hline 25 & BC1_3 & pHSP12 & $\mathrm{BC} 2 \_2$ & OP_TATA_OP & EDD & True \\
\hline 26 & BC1_3 & pHSP12 & BC2_2 & OP_TATA_OP & EDD & True \\
\hline 27 & $\mathrm{BC} 1 \_3$ & pHSP12 & $\mathrm{BC} 2 \_2$ & OP_TATA_OP & EDD & True \\
\hline 28 & BC1_3 & pHSP12 & $\mathrm{BC} 2 \_2$ & OP_TATA_OP & EDD & True \\
\hline 29 & BC1_3 & pHSP12 & $\mathrm{BC} 2 \_2$ & OP_TATA_OP & EDD & True \\
\hline 30 & BC1_3 & pHSP12 & $\mathrm{BC} 2 \_2$ & OP_TATA_OP & DDF & True \\
\hline
\end{tabular}




\begin{tabular}{|c|c|c|c|c|c|c|}
\hline ID & Barcode1 & TF PRO & Barcode2 & fapO insertion scheme & UAS & True/False \\
\hline 31 & BC1_3 & pHSP12 & BC2_2 & OP_TATA_OP & DDF & True \\
\hline 32 & BC1_3 & pHSP12 & $\mathrm{BC} 2 \_2$ & OP_TATA_OP & FBD & True \\
\hline 33 & BC1_3 & pHSP12 & $\mathrm{BC} 2 \_2$ & OP_TATA_OP & BAF & True \\
\hline 34 & BC1_3 & pHSP12 & $\mathrm{BC} 2 \_2$ & OP_TATA_OP & AAC & True \\
\hline 35 & BC1_3 & pHSP12 & $\mathrm{BC} 2 \_3$ & OP_TATA & BDE & True \\
\hline 36 & BC1_3 & pHSP12 & $\mathrm{BC} 2 \_3$ & OP_TATA & BDE & True \\
\hline 37 & BC1_3 & pHSP12 & $\mathrm{BC} 2 \_3$ & OP_TATA & $\mathrm{BCD}$ & True \\
\hline 38 & BC1_3 & pHSP12 & $\mathrm{BC} 2 \_3$ & OP_TATA & $\mathrm{BCD}$ & True \\
\hline 39 & BC1_3 & pHSP12 & $\mathrm{BC} 2 \_3$ & OP_TATA & $\mathrm{BDE}$ & True \\
\hline 40 & BC1_3 & pHSP12 & $\mathrm{BC} 2 \_3$ & OP_TATA & $\mathrm{BDE}$ & True \\
\hline 41 & BC1_3 & pHSP12 & $\mathrm{BC} 2 \_3$ & OP_TATA & FEC & True \\
\hline 42 & BC1_3 & pHSP12 & $\mathrm{BC} 2 \_3$ & OP_TATA & BAC & True \\
\hline 43 & BC1_3 & pHSP12 & $\mathrm{BC} 2 \_3$ & OP_TATA & DCD & True \\
\hline 44 & BC1_3 & pHSP12 & $\mathrm{BC} 2 \_3$ & OP_TATA & EDC & True \\
\hline 45 & BC1_3 & pHSP12 & $\mathrm{BC} 2 \_3$ & OP_TATA & $\mathrm{CDF}$ & True \\
\hline 46 & BC1_3 & pHSP12 & $\mathrm{BC} 2 \_3$ & OP_TATA & AAC & True \\
\hline 47 & BC1_3 & pHSP12 & $\mathrm{BC} 2 \_3$ & OP_TATA & CAD & True \\
\hline 48 & BC1_3 & $p H S P 12$ & $\mathrm{BC} 2 \_3$ & OP_TATA & DAA & True \\
\hline 49 & BC1_3 & pHSP12 & $\mathrm{BC} 2 \_3$ & OP_TATA & FEC & True \\
\hline 50 & BC1_3 & pHSP12 & $\mathrm{BC} 2 \_3$ & OP_TATA & $\mathrm{CDA}$ & True \\
\hline 51 & BC1_3 & pHSP12 & $\mathrm{BC} 2 \_3$ & OP_TATA & $\mathrm{ADF}$ & True \\
\hline 52 & BC1_3 & pHSP12 & BC2_3 & OP_TATA & DAC & True \\
\hline 53 & BC1_3 & pHSP12 & $\mathrm{BC} 2 \_3$ & OP_TATA & $\begin{array}{c}\mathrm{DDA}^{*} \mathrm{~F} \\
\mathrm{CC}\end{array}$ & True \\
\hline 54 & BC1_3 & pHSP12 & BC2_4 & N30_OP & $\mathrm{BDC}$ & True \\
\hline 55 & BC1_3 & pHSP12 & $\mathrm{BC} 2 \_4$ & N30_OP & $\mathrm{BDC}$ & True \\
\hline 56 & $\mathrm{BC} 1 \_3$ & pHSP12 & $\mathrm{BC} 2 \_4$ & N30_OP & CAD & True \\
\hline 57 & $\mathrm{BC} 1 \_3$ & pHSP12 & $\mathrm{BC} 2 \_4$ & N30_OP & DBA & True \\
\hline 58 & BC1_4 & $p E X G 1$ & $\mathrm{BC} 2 \_1$ & TATA_OP & AAF & True \\
\hline 59 & BC1_4 & $p E X G 1$ & $\mathrm{BC} 2 \_1$ & TATA_OP & DEC & True \\
\hline 60 & BC1_4 & $p E X G 1$ & $\mathrm{BC} 2 \_1$ & TATA_OP & $\mathrm{ADA}$ & True \\
\hline 61 & BC1_4 & $p E X G 1$ & $\mathrm{BC} 2 \_1$ & TATA_OP & FEC & True \\
\hline 62 & BC1_4 & $p E X G 1$ & $\mathrm{BC} 2 \_1$ & TATA_OP & FEC & True \\
\hline 63 & BC1_4 & $p E X G 1$ & BC2_1 & TATA_OP & DBD & True \\
\hline
\end{tabular}




\begin{tabular}{|c|c|c|c|c|c|c|}
\hline ID & Barcode1 & TF PRO & Barcode2 & fapO insertion scheme & UAS & True/False \\
\hline 64 & BC1_4 & $p E X G 1$ & $\mathrm{BC} 2 \_2$ & OP_TATA_OP & $* \mathrm{AC}$ & True \\
\hline 65 & BC1_4 & $p E X G 1$ & $\mathrm{BC} 2 \_2$ & OP_TATA_OP & BFD & True \\
\hline 66 & BC1_4 & $p E X G 1$ & $\mathrm{BC} 2 \_2$ & OP_TATA_OP & $\mathrm{CBC}$ & True \\
\hline 67 & BC1_4 & $p E X G 1$ & $\mathrm{BC} 2 \_2$ & OP_TATA_OP & BFA & True \\
\hline 68 & BC1_4 & $p E X G 1$ & $\mathrm{BC} 2 \_2$ & OP_TATA_OP & EAC & True \\
\hline 69 & BC1_4 & $p E X G 1$ & $\mathrm{BC} 2 \_2$ & OP_TATA_OP & EDC & True \\
\hline 70 & BC1_4 & $p E X G 1$ & $\mathrm{BC} 2 \_2$ & OP_TATA_OP & $\mathrm{BCC}$ & True \\
\hline 71 & BC1_4 & $p E X G 1$ & $\mathrm{BC} 2 \_2$ & OP_TATA_OP & $\mathrm{BBC}$ & True \\
\hline 72 & BC1_4 & $p E X G 1$ & $\mathrm{BC} 2 \_3$ & OP_TATA & BEE & True \\
\hline 73 & BC1_4 & $p E X G 1$ & $\mathrm{BC} 2 \_3$ & OP_TATA & BEE & True \\
\hline 74 & BC1_4 & $p E X G 1$ & $\mathrm{BC} 2 \_3$ & OP_TATA & EDF & True \\
\hline 75 & BC1_4 & $p E X G 1$ & $\mathrm{BC} 2 \_3$ & OP_TATA & EDF & True \\
\hline 76 & BC1_4 & $p E X G 1$ & $\mathrm{BC} 2 \_3$ & OP_TATA & EBF & True \\
\hline 77 & BC1_4 & $p E X G 1$ & $\mathrm{BC} 2 \_3$ & OP_TATA & EDC & True \\
\hline 78 & BC1_4 & $p E X G 1$ & $\mathrm{BC} 2 \_3$ & OP_TATA & $\mathrm{AEC}$ & True \\
\hline 79 & BC1_4 & $p E X G 1$ & $\mathrm{BC} 2 \_3$ & OP_TATA & ADD & True \\
\hline 80 & BC1_4 & $p E X G 1$ & $\mathrm{BC} 2 \_3$ & OP_TATA & EAC & True \\
\hline 81 & BC1_4 & $p E X G 1$ & $\mathrm{BC} 2 \_3$ & OP_TATA & FFF & True \\
\hline 82 & BC1_4 & $p E X G 1$ & $\mathrm{BC} 2 \_3$ & OP_TATA & DDF & True \\
\hline 83 & BC1_4 & $p E X G 1$ & $\mathrm{BC} 2 \_3$ & OP_TATA & $\mathrm{BBF}$ & True \\
\hline 84 & BC1_4 & $p E X G 1$ & BC2_4 & N30_OP & $\mathrm{CCC}$ & True \\
\hline 85 & BC1_4 & $p E X G 1$ & $\mathrm{BC} 2 \_4$ & N30_OP & $\mathrm{CCC}$ & True \\
\hline 86 & BC1_4 & $p E X G 1$ & $\mathrm{BC} 2 \_4$ & N30_OP & BAC & True \\
\hline 87 & BC1_4 & $p E X G 1$ & BC2_4 & N30_OP & $\begin{array}{c}\mathrm{FFF}^{*} \mathrm{D} \\
\mathrm{BB} * \mathrm{DE} \\
\mathrm{D}\end{array}$ & True \\
\hline 88 & BC1_4 & $p E X G 1$ & BC2_4 & N30_OP & $\mathrm{CDC}$ & True \\
\hline 89 & BC1_5 & $p C Y C 1$ & $\mathrm{BC} 2 \_1$ & TATA_OP & BAF & True \\
\hline 90 & $\mathrm{BC} 1 \_5$ & $p C Y C 1$ & $\mathrm{BC} 2 \_1$ & TATA_OP & FAF & True \\
\hline 91 & BC1_5 & $p C Y C 1$ & $\mathrm{BC} 2 \_1$ & TATA_OP & EAC & True \\
\hline 92 & BC1_5 & $p C Y C 1$ & $\mathrm{BC} 2 \_1$ & TATA_OP & EED & True \\
\hline 93 & BC1_5 & $p C Y C 1$ & BC2_1 & TATA_OP & $\mathrm{CCC}$ & True \\
\hline 94 & BC1_5 & $p C Y C 1$ & BC2_1 & TATA_OP & $\mathrm{CCF}$ & True \\
\hline 95 & BC1_5 & $p C Y C 1$ & BC2_2 & OP_TATA_OP & EDD & True \\
\hline
\end{tabular}




\begin{tabular}{|c|c|c|c|c|c|c|}
\hline ID & Barcode1 & TF PRO & Barcode2 & fapO insertion scheme & UAS & True/False \\
\hline 96 & BC1_5 & $p C Y C 1$ & $\mathrm{BC} 2 \_2$ & OP_TATA_OP & $\mathrm{BBA}$ & True \\
\hline 97 & BC1_5 & $p C Y C 1$ & BC2_2 & OP_TATA_OP & DCC & True \\
\hline 98 & BC1_5 & $p C Y C 1$ & $\mathrm{BC} 2 \_3$ & OP_TATA & CFD & True \\
\hline 99 & BC1_5 & $p C Y C 1$ & $\mathrm{BC} 2 \_3$ & OP_TATA & DBF & True \\
\hline 100 & BC1_5 & $p C Y C 1$ & $\mathrm{BC} 2 \_3$ & OP_TATA & DED & True \\
\hline 101 & BC1_5 & $p C Y C 1$ & $\mathrm{BC} 2 \_3$ & OP_TATA & CFA & True \\
\hline 102 & BC1_5 & $p C Y C 1$ & BC2_3 & OP_TATA & $\mathrm{BEC}$ & True \\
\hline 103 & BC1_5 & $p C Y C 1$ & $\mathrm{BC} 2 \_3$ & OP_TATA & FDF & True \\
\hline 104 & BC1_5 & $p C Y C 1$ & $\mathrm{BC} 2 \_3$ & OP_TATA & $\mathrm{ACC}$ & True \\
\hline 105 & BC1_5 & $p C Y C 1$ & $\mathrm{BC} 2 \_3$ & OP_TATA & CAC & True \\
\hline 106 & BC1_5 & $p C Y C 1$ & $\mathrm{BC} 2 \_3$ & OP_TATA & $\mathrm{BEC}$ & True \\
\hline 107 & BC1_5 & $p C Y C 1$ & $\mathrm{BC} 2 \_4$ & N30_OP & DBF & True \\
\hline 108 & BC1_5 & $p C Y C 1$ & $\mathrm{BC} 2 \_4$ & N30_OP & FAC & True \\
\hline 109 & BC1_5 & $p C Y C 1$ & $\mathrm{BC} 2 \_4$ & N30_OP & $\mathrm{EBC}$ & True \\
\hline 110 & BC1_5 & $p C Y C 1$ & BC2_4 & N30_OP & BAD & True \\
\hline 111 & BC1_5 & $p C Y C 1$ & BC2_4 & N30_OP & $\mathrm{BBF}$ & True \\
\hline 112 & BC1_5 & $p C Y C 1$ & BC2_4 & N30_OP & $\mathrm{F}^{*} \mathrm{D}$ & True \\
\hline 113 & BC1_6 & $p U L I 1$ & BC2_1 & TATA_OP & $\mathrm{CBA}$ & True \\
\hline 114 & BC1_6 & $p U L I 1$ & BC2_1 & TATA_OP & $\mathrm{BCF}$ & True \\
\hline 115 & BC1_6 & $p U L I 1$ & BC2_1 & TATA_OP & DCA & True \\
\hline 116 & BC1_6 & $p U L I 1$ & BC2_1 & TATA_OP & BAF & True \\
\hline 117 & BC1_6 & $p U L I 1$ & BC2_2 & OP_TATA_OP & $\mathrm{BDD}$ & True \\
\hline 118 & BC1_6 & $p U L I 1$ & BC2_2 & OP_TATA_OP & FAD & True \\
\hline 119 & BC1_6 & $p U L I 1$ & BC2_2 & OP_TATA_OP & EDF & True \\
\hline 120 & BC1_6 & $p U L I 1$ & BC2_2 & OP_TATA_OP & $\mathrm{CDC}$ & True \\
\hline 121 & BC1_6 & $p U L I 1$ & BC2_2 & OP_TATA_OP & $\mathrm{ADF}$ & True \\
\hline 122 & BC1_6 & $p U L I 1$ & BC2_2 & OP_TATA_OP & DAC & True \\
\hline 123 & BC1_6 & $p U L I 1$ & BC2_2 & OP_TATA_OP & $\mathrm{A} * \mathrm{~A}$ & True \\
\hline 124 & BC1_6 & $p U L I 1$ & $\mathrm{BC} 2 \_2$ & OP_TATA_OP & $\mathrm{C}^{*} \mathrm{C}$ & True \\
\hline 125 & BC1_6 & $p U L I 1$ & $\mathrm{BC} 2 \_3$ & OP_TATA & $\mathrm{CFC}$ & True \\
\hline 126 & BC1_6 & $p U L I 1$ & $\mathrm{BC} 2 \_3$ & OP_TATA & $\mathrm{BFC}$ & True \\
\hline 127 & BC1_6 & $p U L I 1$ & BC2_3 & OP_TATA & FBF & True \\
\hline 128 & BC1_6 & $p U L I 1$ & $\mathrm{BC} 2 \_3$ & OP_TATA & $* \mathrm{DC}$ & True \\
\hline
\end{tabular}




\begin{tabular}{lcccccc}
\hline ID & Barcode1 & TF PRO & Barcode2 & fapO insertion scheme & UAS & True/False \\
\hline 129 & BC1_6 & $p U L I 1$ & BC2_3 & OP_TATA & EB* & True \\
130 & BC1_6 & $p U L I 1$ & BC2_4 & N30_OP & ADC & True \\
131 & BC1_6 & $p U L I 1$ & BC2_4 & N30_OP & FAD & True \\
132 & BC1_6 & $p U L I 1$ & BC2_4 & N30_OP & DCF & True \\
133 & BC1_6 & $p U L I 1$ & BC2_4 & N30_OP & BBC & True \\
\hline$*$
\end{tabular}


Table S4 The distribution of 2632 FapR-fapO based malonyl-CoA biosensors characterized by FACS-seq

\begin{tabular}{ccccccc}
\hline & N30_OP & OP_TATA & OP_TATA_OP & TATA_OP & Total & Ratio \\
\hline$p G P D$ & 13 & 43 & 13 & 11 & 80 & 0.093 \\
$p E N O 2$ & 48 & 73 & 38 & 60 & 219 & 0.253 \\
$p H S P 12$ & 135 & 147 & 146 & 148 & 576 & 0.667 \\
$p E X G 1$ & 153 & 140 & 133 & 137 & 563 & 0.652 \\
$p C Y C 1$ & 162 & 158 & 146 & 143 & 609 & 0.705 \\
$p U L I 1$ & 150 & 157 & 138 & 140 & 585 & 0.677 \\
Total & 661 & 718 & 614 & 639 & 2632 & 0.508 \\
\hline
\end{tabular}


Table S5 The Gini coefficient of $\sum_{P 2}^{P 9} r_{j, s_{k}}$ distribution in Lib0A (FapR-fapO metabolite biosensor library with $0 \mathrm{mg} / \mathrm{L}$ cerulenin addition)

\begin{tabular}{cccccc}
\hline & TATA_OP & OP_TATA & $\begin{array}{c}\text { OP_TATA_O } \\
\text { P }\end{array}$ & N30_OP & 总计 \\
\hline$p G P D$ & 0.973 & 0.991 & 0.951 & 0.980 & 0.990 \\
$p E N O 2$ & 0.865 & 0.978 & 0.897 & 0.928 & 0.961 \\
$p H S P 12$ & 0.820 & 0.787 & 0.731 & 0.719 & 0.776 \\
$p E X G 1$ & 0.867 & 0.704 & 0.628 & 0.757 & 0.787 \\
$p C Y C 1$ & 0.679 & 0.689 & 0.648 & 0.643 & 0.699 \\
$p U L I 1$ & 0.707 & 0.682 & 0.720 & 0.763 & 0.740 \\
\hline
\end{tabular}


Table S7 Strains and plasmids used in this work

\begin{tabular}{|c|c|c|}
\hline Strains/plasmids & Characteristics & Sources \\
\hline \multicolumn{3}{|l|}{ Strains } \\
\hline E. coli $\mathrm{DH} 10 \mathrm{~B}$ & & Biomed \\
\hline E. coli DB3.1 & & Gift from DaiLab \\
\hline BY4700 & MATa ura $3 \Delta 0$ & Gift from DaiLab \\
\hline \multicolumn{3}{|l|}{ Plasmids } \\
\hline $\begin{array}{c}\text { HCKan_P_pGPD, } \\
\text { HCKan_P_pENO2, HCKan_pHSP12, } \\
\text { HCKan_P_pEXG1, } \\
\text { HCKan_P_pCYC1, } \\
\text { HCKan_P_pULI1 }\end{array}$ & Standardized promoters, $\mathrm{Km}^{\mathrm{R}}$ & 1 \\
\hline pKMV_yFapR & $\begin{array}{c}\text { Condon optimized FapR } \\
\text { gene, } \mathrm{Km}^{\mathrm{R}}\end{array}$ & Wuxi Qinglan \\
\hline РOT2, РОT3 & POT vector, Amp ${ }^{R}$ & 1 \\
\hline New_HCKan_T_tCYC1 & $\begin{array}{l}\text { Standardized terminator } \mathrm{tCYC} 1 \\
\text { for the construction of POTB, } \\
\qquad \mathrm{Km}^{\mathrm{R}}\end{array}$ & This study \\
\hline $\begin{array}{l}\text { POT2-pGPD-FapR-tCYC1-BC1_1, } \\
\text { POT2-pENO2-FapR-tCYC1-BC1_2, } \\
\text { POT2-pHSP12-FapR-tCYC1-BC1_3, } \\
\text { POT2-pEXG1-FapR-tCYC1-BC1_4, } \\
\text { POT2-pCYC1-FapR-tCYC1-BC1_5, } \\
\text { POT2-pULI1-FapR-tCYC1-BC1_6 }\end{array}$ & POTB contructed, $\mathrm{Amp}^{\mathrm{R}}$ & This study \\
\hline $\mathrm{HC}-\mathrm{AcV}$ & Accept vector in Step1, $\mathrm{Km}^{\mathrm{R}}$ & This study \\
\hline $\begin{array}{c}\text { HC-AcV -pGPD-FapR-tCYC1- } \\
\text { BC1_1, HC-AcV -pENO2-FapR- } \\
\text { tCYC1-BC1_2, HC-AcV -pHSP12- } \\
\text { FapR-tCYC1-BC1_3, HC-AcV - } \\
\text { pEXG1-FapR-tCYC1-BC1_4, HC- } \\
\text { AcV -pCYC1-FapR-tCYC1-BC1_5, } \\
\text { HC-AcV -pULI1-FapR-tCYC1- } \\
\text { BC1 6 }\end{array}$ & $\begin{array}{c}\text { HC-AcV-Pro-FapR-tCYC1- } \\
\text { BC1, } \mathrm{Km}^{\mathrm{R}}\end{array}$ & This study \\
\hline $\begin{array}{l}\text { pKMV-FP1, pKMV-FP2, } \\
\text { pKMV-FP3, pKMV-FP4 }\end{array}$ & $\begin{array}{l}\text { Synthetic promoters with fapO, } \\
\qquad \mathrm{Km}^{\mathrm{R}}\end{array}$ & Wuxi Qinglan \\
\hline HCKan_O_YPet & Standardized YPet, $\mathrm{Km}^{\mathrm{R}}$ & This study \\
\hline HCKan_T_tPGK1 & $\begin{array}{l}\text { Standardized terminator } \\
\text { tPGK1, } \mathrm{Km}^{\mathrm{R}}\end{array}$ & This study \\
\hline $\begin{array}{l}\text { POT3-FP1-YPet-tPGK1, } \\
\text { POT3-FP2-YPet-tPGK1, } \\
\text { POT3-FP3-YPet-tPGK1, } \\
\text { POT3-FP4-YPet-tPGK1 }\end{array}$ & $\begin{array}{l}\text { POT3-Synthtic promoter-YPet- } \\
\text { tPGK1, Amp }{ }^{R}\end{array}$ & This study \\
\hline $\begin{array}{c}\text { Assembled-Rev-POT3-ccdB-FP1- } \\
\text { YPet-tPGK1, Assembled-Rev-POT3- } \\
\text { ccdB-FP2-YPet-tPGK1, Assembled- } \\
\text { Rev-POT3-ccdB-FP3-YPet-tPGK1, } \\
\text { Assembled-Rev-POT3-ccdB-FP4- } \\
\text { YPet-tPGK1 }\end{array}$ & $\begin{array}{l}\text { Vector for the insertion of } \\
\text { UAS, Amp }{ }^{R}\end{array}$ & This study \\
\hline AC-Reporter & $\begin{array}{l}\text { Accept vector in Step 5, } \\
\qquad \mathrm{Amp}^{\mathrm{R}}, \mathrm{DB} 3.1\end{array}$ & This study \\
\hline POT1-pTEF2-mCherry-tADH1 & $\begin{array}{l}\text { Positive control plasmids for } \\
\text { mCherry, Amp }{ }^{\mathrm{R}}\end{array}$ & This study \\
\hline
\end{tabular}


POT1-pCYC1-YPet-tPGK1

pGPD-TATA_OP-UAS_BBC
Positive control plasmids for YPet, $A m p^{R}$

Dual positive control plasmids for YPet and mCherry, Amp ${ }^{\mathrm{R}}$
This study

This study 
Table S8 Primers and other oligonucleotides used in this work

\begin{tabular}{|c|c|c|}
\hline Primers & Sequences & Usages \\
\hline $\mathrm{HC}-\mathrm{AcV}-1$ & gagtcCGTCTCgACCTaGAGACCgaattcgcgg & $\begin{array}{l}\text { Forward primer to clone the } \\
\text { fragment1 from } \mathrm{HCK} \text { an_O to } \\
\text { construct } \mathrm{HC}-\mathrm{AcV}\end{array}$ \\
\hline $\mathrm{HC}-\mathrm{AcV}-2$ & cagtCGTCTCgGCCTaGAGACCctgca & $\begin{array}{l}\text { Reverse primer to clone the fragment1 } \\
\text { from HCKan_O to construct } \mathrm{HC}-\mathrm{AcV}\end{array}$ \\
\hline $\mathrm{HC}-\mathrm{AcV}-3$ & tgcagGGTCTCtAGGCcGAGACGactg & $\begin{array}{c}\text { Forward primer to clone the } \\
\text { fragment } 2 \text { from HCKan_O to } \\
\text { construct } \mathrm{HC}-\mathrm{AcV}\end{array}$ \\
\hline $\mathrm{HC}-\mathrm{AcV}-4$ & ccgcgaattcGGTCTCtAGGTcGAGACGgactc & $\begin{array}{l}\text { Reverse primer to clone the fragment2 } \\
\text { from HCKan_O to construct } \mathrm{HC}-\mathrm{AcV}\end{array}$ \\
\hline FapR-test01 & TAGCTTGATCATCCAATTCCAAATCA & $\begin{array}{l}\text { Primer } 1 \text { used for sequencing in } \\
\text { trackable library sampling }\end{array}$ \\
\hline YPet-test01 & gTCAgCAgTgATgTAAACATTgTg & $\begin{array}{l}\text { Primer2 used for sequencing in } \\
\text { trackable library sampling }\end{array}$ \\
\hline HU-03 & tcGGTCTCtCGCCcGAGACGgactccagcg & $\begin{array}{c}\text { Forward primer to amplify the } \\
\text { HCKan_U fragment from HCKan_O }\end{array}$ \\
\hline HU-04 & gctgcagGGTCTCtTTAAcGAGACGactgacca & $\begin{array}{l}\text { Reverse primer to amplify the } \\
\text { HCKan_U fragment from HCKan_O }\end{array}$ \\
\hline $\mathrm{BC} 001 \mathrm{~F}$ & $\begin{array}{c}\text { AAGTAGGCTGGTCTTCGCTCGCATGCG } \\
\text { GAAGACAGTGAGAAAACCC }\end{array}$ & $\begin{array}{l}\text { Oligonucleotide used for annealed } \\
\text { linker to construct POT2-pGPD- } \\
\text { FapR-tCYC1-BC1_1 }\end{array}$ \\
\hline $\mathrm{BC} 001 \mathrm{R}$ & $\begin{array}{c}\text { GAGGGGGTTTTCTCACTGTCTTCCGCAT } \\
\text { GCGAGCGAAGACCAGCCT }\end{array}$ & $\begin{array}{l}\text { Oligonucleotide used for annealed } \\
\text { linker to construct POT2-pGPD- } \\
\text { FapR-tCYC1-BC1_1 }\end{array}$ \\
\hline $\mathrm{BC} 002 \mathrm{~F}$ & $\begin{array}{c}\text { AAGTAGGCTGGTCTTCGCTCGCATGCG } \\
\text { GAAGACAGTGAGAAACAGT }\end{array}$ & $\begin{array}{l}\text { Oligonucleotide used for annealed } \\
\text { linker to construct POT2-pENO2- } \\
\text { FapR-tCYC1-BC1_2 }\end{array}$ \\
\hline $\mathrm{BC} 002 \mathrm{R}$ & $\begin{array}{c}\text { GAGGACTGTTTCTCACTGTCTTCCGCAT } \\
\text { GCGAGCGAAGACCAGCCT }\end{array}$ & $\begin{array}{l}\text { Oligonucleotide used for annealed } \\
\text { linker to construct POT2-pENO2- } \\
\text { FapR-tCYC1-BC1_2 }\end{array}$ \\
\hline $\mathrm{BC} 003 \mathrm{~F}$ & $\begin{array}{c}\text { AAGTAGGCTGGTCTTCGCTCGCATGCG } \\
\text { GAAGACAGTGAGAAACTAA }\end{array}$ & $\begin{array}{l}\text { Oligonucleotide used for annealed } \\
\text { linker to construct POT2-pHSP12- } \\
\text { FapR-tCYC1-BC1_3 }\end{array}$ \\
\hline $\mathrm{BC} 003 \mathrm{R}$ & $\begin{array}{c}\text { GAGGTTAGTTTCTCACTGTCTTCCGCAT } \\
\text { GCGAGCGAAGACCAGCCT }\end{array}$ & $\begin{array}{l}\text { Oligonucleotide used for annealed } \\
\text { linker to construct POT2-pHSP12- } \\
\text { FapR-tCYC1-BC1_3 }\end{array}$ \\
\hline $\mathrm{BC} 004 \mathrm{~F}$ & $\begin{array}{c}\text { AAGTAGGCTGGTCTTCGCTCGCATGCG } \\
\text { GAAGACAGTGAGAAAGATC }\end{array}$ & $\begin{array}{l}\text { Oligonucleotide used for annealed } \\
\text { linker to construct POT2-pEXG1- } \\
\text { FapR-tCYC1-BC1_4 }\end{array}$ \\
\hline $\mathrm{BC} 004 \mathrm{R}$ & $\begin{array}{c}\text { GAGGGATCTTTCTCACTGTCTTCCGCAT } \\
\text { GCGAGCGAAGACCAGCCT }\end{array}$ & $\begin{array}{l}\text { Oligonucleotide used for annealed } \\
\text { linker to construct POT2-pEXG1- } \\
\text { FapR-tCYC1-BC1_4 }\end{array}$ \\
\hline $\mathrm{BC} 005 \mathrm{~F}$ & $\begin{array}{c}\text { AAGTAGGCTGGTCTTCGCTCGCATGCG } \\
\text { GAAGACAGTGAGAAAGCGG }\end{array}$ & $\begin{array}{l}\text { Oligonucleotide used for annealed } \\
\text { linker to construct POT2-pCYC1- } \\
\text { FapR-tCYC1-BC1_5 }\end{array}$ \\
\hline $\mathrm{BC} 005 \mathrm{R}$ & $\begin{array}{c}\text { GAGGCCGCTTTCTCACTGTCTTCCGCAT } \\
\text { GCGAGCGAAGACCAGCCT }\end{array}$ & $\begin{array}{l}\text { Oligonucleotide used for annealed } \\
\text { linker to construct POT2-pCYC1- } \\
\text { FapR-tCYC1-BC1_5 }\end{array}$ \\
\hline $\mathrm{BC} 006 \mathrm{~F}$ & $\begin{array}{c}\text { AAGTAGGCTGGTCTTCGCTCGCATGCG } \\
\text { GAAGACAGTGAGAACCCAG }\end{array}$ & $\begin{array}{l}\text { Oligonucleotide used for annealed } \\
\text { linker to construct POT2-pULI1- } \\
\text { FapR-tCYC1-BC1_6 }\end{array}$ \\
\hline $\mathrm{BC} 006 \mathrm{R}$ & $\begin{array}{c}\text { GAGGCTGGGTTCTCACTGTCTTCCGCA } \\
\text { TGCGAGCGAAGACCAGCCT }\end{array}$ & $\begin{array}{c}\text { Oligonucleotide used for annealed } \\
\text { linker to construct POT2-pULI1- } \\
\text { FapR-tCYC1-BC1_6 }\end{array}$ \\
\hline
\end{tabular}




\begin{tabular}{|c|c|c|}
\hline NGS_01 & $\begin{array}{c}\text { CAAATACGCTTGATTAGAATATTACAA } \\
\text { GTTAATTAA }\end{array}$ & $\begin{array}{l}\text { Forward primer to amplify the } \\
\text { barcodes region for plasmids } \\
\text { extracted from cells sorted in P2 }\end{array}$ \\
\hline NGS_02 & TCACTGCtcgtctaccetatgaacatat & $\begin{array}{l}\text { Reverse primer to amplify the } \\
\text { barcodes region for plasmids } \\
\text { extracted from cells sorted in P2 }\end{array}$ \\
\hline NGS_03 & $\begin{array}{l}\text { CAACCGCGCTTGATTAGAATATTACAA } \\
\text { GTTAATTAA }\end{array}$ & $\begin{array}{l}\text { Forward primer to amplify the } \\
\text { barcodes region for plasmids } \\
\text { extracted from cells sorted in } \mathrm{P} 3\end{array}$ \\
\hline NGS_04 & TCAGAGAtcgtctaccctatgaacatat & $\begin{array}{l}\text { Reverse primer to amplify the } \\
\text { barcodes region for plasmids } \\
\text { extracted from cells sorted in } \mathrm{P} 3\end{array}$ \\
\hline NGS_05 & $\begin{array}{l}\text { CAAGTCTGCTTGATTAGAATATTACAA } \\
\text { GTTAATTAA }\end{array}$ & $\begin{array}{l}\text { Forward primer to amplify the } \\
\text { barcodes region for plasmids } \\
\text { extracted from cells sorted in } \mathrm{P} 4\end{array}$ \\
\hline NGS_06 & TCCAAGCtcgtctaccetatgaacatat & $\begin{array}{l}\text { Reverse primer to amplify the } \\
\text { barcodes region for plasmids } \\
\text { extracted from cells sorted in } \mathrm{P} 4\end{array}$ \\
\hline NGS_07 & $\begin{array}{l}\text { CAATGGAGCTTGATTAGAATATTACAA } \\
\text { GTTAATTAA }\end{array}$ & $\begin{array}{l}\text { Forward primer to amplify the } \\
\text { barcodes region for plasmids } \\
\text { extracted from cells sorted in P5 }\end{array}$ \\
\hline NGS_08 & TCCCCGAtcgtctaccetatgaacatat & $\begin{array}{l}\text { Reverse primer to amplify the } \\
\text { barcodes region for plasmids } \\
\text { extracted from cells sorted in P5 }\end{array}$ \\
\hline NGS_09 & $\begin{array}{l}\text { CACAATTGCTTGATTAGAATATTACAA } \\
\text { GTTAATTAA }\end{array}$ & $\begin{array}{l}\text { Forward primer to amplify the } \\
\text { barcodes region for plasmids } \\
\text { extracted from cells sorted in P6 }\end{array}$ \\
\hline NGS_10 & TCCGCAGtcgtctaccetatgaacatat & $\begin{array}{l}\text { Reverse primer to amplify the } \\
\text { barcodes region for plasmids } \\
\text { extracted from cells sorted in P6 }\end{array}$ \\
\hline NGS_11 & $\begin{array}{l}\text { CACAGAGGCTTGATTAGAATATTACAA } \\
\text { GTTAATTAA }\end{array}$ & $\begin{array}{l}\text { Forward primer to amplify the } \\
\text { barcodes region for plasmids } \\
\text { extracted from cells sorted in } \mathrm{P} 7\end{array}$ \\
\hline NGS_12 & TCCTTTAtcgtctaccetatgaacatat & $\begin{array}{l}\text { Reverse primer to amplify the } \\
\text { barcodes region for plasmids } \\
\text { extracted from cells sorted in P7 }\end{array}$ \\
\hline NGS_13 & $\begin{array}{l}\text { CACCAAAGCTTGATTAGAATATTACAA } \\
\text { GTTAATTAA }\end{array}$ & $\begin{array}{l}\text { Forward primer to amplify the } \\
\text { barcodes region for plasmids } \\
\text { extracted from cells sorted in P8 }\end{array}$ \\
\hline NGS_14 & TCGATTGtcgtctaccctatgaacatat & $\begin{array}{l}\text { Reverse primer to amplify the } \\
\text { barcodes region for plasmids } \\
\text { extracted from cells sorted in P8 }\end{array}$ \\
\hline NGS_15 & $\begin{array}{l}\text { CACTCGGGCTTGATTAGAATATTACAA } \\
\text { GTTAATTAA }\end{array}$ & $\begin{array}{l}\text { Forward primer to amplify the } \\
\text { barcodes region for plasmids } \\
\text { extracted from cells sorted in P9 }\end{array}$ \\
\hline NGS_16 & TCGCGATtcgtctaccetatgaacatat & $\begin{array}{l}\text { Reverse primer to amplify the } \\
\text { barcodes region for plasmids } \\
\text { extracted from cells sorted in P9 }\end{array}$ \\
\hline
\end{tabular}


Table S9 The prefix and suffix sequences of the genetic elements used in POTB assembly

\begin{tabular}{ccc}
\hline Genetic element & Prefixes & Suffixes \\
\hline HCKan_P_PRO & GGCT & GATG \\
pKMV_yFapR & GATG & TAGC \\
HCKan_T_tCYC1 & TAGC & CCTC \\
POT2 & GGCT & CCTC \\
New_HCKan_T_tCYC1 & TAGC & AAGT \\
Linker & AAGT & CCTC \\
\hline
\end{tabular}


Table S10 The promoters that drive the expression of FapR and their corresponding molecular barcode Barcode1

\begin{tabular}{|c|c|c|c|c|c|c|}
\hline ID & $\begin{array}{c}\text { Standard } \\
\text { name }\end{array}$ & $\begin{array}{l}\text { System } \\
\text { name }\end{array}$ & Length & $\begin{array}{l}\text { Relative } \\
\text { activity }\end{array}$ & $\begin{array}{l}\text { Linlog } \\
\text { activity }\end{array}$ & Barcode1(BC1) \\
\hline BC1_1 & $p G P D$ & $p Y G R 192 C$ & $667 \mathrm{bp}$ & 54.89 & 1.00 & AAAACCC \\
\hline BC1_2 & pENO2 & pYHR174W & $500 \mathrm{bp}$ & 39.14 & 0.87 & AAACAGT \\
\hline $\mathrm{BC} 1 \_3$ & pHSP12 & pYFL014W & $500 \mathrm{bp}$ & 17.34 & 0.54 & AAACTAA \\
\hline BC1_4 & $p E X G 1$ & $p Y L R 300 W$ & $500 \mathrm{bp}$ & 8.39 & 0.25 & AAAGATC \\
\hline BC1_5 & $p C Y C 1$ & pYJR048W & $295 \mathrm{bp}$ & 1.00 & -0.59 & AAAGCGG \\
\hline BC1_6 & $p U L I 1$ & pYFR026C & $500 \mathrm{bp}$ & 0.36 & -1.00 & AACCCAG \\
\hline
\end{tabular}


Table S11 The reaction system to construct standard transcription unit PRO-ORF-

TER-BC1 (POTB)

\begin{tabular}{cc}
\hline Reagent & Volume $(\mu \mathrm{L})$ \\
\hline 10 $\times$ FastDigest Buffer & 1.0 \\
25mM ATP & 0.2 \\
DTT (20mM) & 0.5 \\
Esp3I (BsmBI, Thermo Scientific, FD0454) & 0.5 \\
T4 DNA Ligase (5 U/ $\mu$ L) (Thermo Scientific, EL0011) & 0.5 \\
POT2(Vector) & $50 \mathrm{ng}$ \\
HCKan_P_Pro & $50 \mathrm{ng}$ \\
pKMV1-yFapR & $100 \mathrm{ng}$ \\
New_HCKan_T_tCYC1 & $50 \mathrm{ng}$ \\
Annealed Linker & 0.5 \\
ddH ${ }_{2} \mathrm{O}$ & Up to 10 \\
\hline
\end{tabular}


Table S12 The GoldenGate reaction program based on FastDigest enzyme

\begin{tabular}{cccc}
\hline GoldenGate Step & Temperature & Time & Cycles \\
\hline Pre-digestion & $37^{\circ} \mathrm{C}$ & $25 \mathrm{~min}$ & 1 \\
Digestion & $37{ }^{\circ} \mathrm{C}$ & $5 \mathrm{~min}$ & 10 \\
Ligation & $22^{\circ} \mathrm{C}$ & $10 \mathrm{~min}$ & \\
Final-digestion & $37{ }^{\circ} \mathrm{C}$ & $5 \mathrm{~min}$ & 1 \\
Heat shock & $65^{\circ} \mathrm{C}$ & $10 \mathrm{~min}$ & 1 \\
Store & $4{ }^{\circ} \mathrm{C}$ & forever & 1 \\
\hline
\end{tabular}


Table S13 The reaction system to transform the POTB fragment into HC-AcV

\begin{tabular}{cc}
\hline Reagent & Volume $(\mu \mathrm{L})$ \\
\hline 10 $\times$ FastDigest Buffer & 1 \\
25mM ATP & 0.2 \\
Eco31I (BsaI, Thermo Scientific, FD0293) & 0.5 \\
T4 DNA Ligase $(5 \mathrm{U} / \mu \mathrm{L})$ (Thermo Scientific, EL0011) & 0.5 \\
$\mathrm{HC}-\mathrm{AcV}$ (Vector) & $10 \mathrm{ng}$ \\
POTB & $50 \mathrm{ng}$ \\
$\mathrm{ddH}_{2} \mathrm{O}$ & $\mathrm{Up}$ to 10 \\
\hline
\end{tabular}


Table S14 The operator insertion scheme in the core element of the synthetic minimal yeast promoters and their corresponding Barcode2

\begin{tabular}{cccc}
\hline ID & Barcode2 & fapO insertion scheme & Synthetic promoter \\
\hline BC2_1 & AAGCATA & TATA_OP & pKMV-FP1 \\
BC2_2 & AAGCGAC & OP_TATA_OP & pKMV-FP2 \\
BC2_3 & AAGGCCA & OP_TATA & pKMV-FP3 \\
BC2_4 & AAGTGGT & N30_OP & pKMV-FP4 \\
\hline
\end{tabular}


Table S15 The reaction system to assemble the HCKan-UAS library

\begin{tabular}{cc}
\hline Reagent & Volume $(\mu \mathrm{L})$ \\
\hline HCKan_U fragment with BsaI digestion & $\sim 400 \mathrm{ng}$ \\
UAS_Linker mixture & 8 \\
T4 DNA Ligase $(5 \mathrm{U} / \mu \mathrm{L})$ (Thermo Scientific, & 8 \\
EL0011) & 40 \\
$10 \times$ T4 DNA Ligase Buffer & 2 \\
$100 \mathrm{mM}_{\text {ATP }}$ & Up to 400 \\
\hline
\end{tabular}


Table S16 The reaction system to transform the UAS sequence into ccdB-YPettPGK1vector

\begin{tabular}{cc}
\hline Reagent & Volume $(\mu \mathrm{L})$ \\
\hline $10 \times$ FastDigest Buffer & 5.0 \\
25 mM ATP & 1.0 \\
DTT (20 mM) & 2.5 \\
Esp3I (BsmBI, Thermo Scientific, FD0454) & 2.5 \\
T4 DNA Ligase $(5$ U/ $\mu$ L) (Thermo Scientific, EL0011) & 2.5 \\
ccdB-YPet-tPGK1 (vector) & $\sim 250 \mathrm{ng}$ \\
HCKan-UAS library & 2.5 \\
ddH ${ }_{2} \mathrm{O}$ & Up to 50 \\
\hline
\end{tabular}


Table S17 The digestion reaction system for HC-AcV-POTB/ UAS-YPet-tPGK1

\begin{tabular}{cc}
\hline Reagent & Volume $(\mu \mathrm{L})$ \\
\hline $10 \times$ Fast Digest & 4 \\
HC-AcV-POTB/ UAS-YPet-tPGK1 & $\sim 2 \mu \mathrm{g}$ \\
Fast Digest Enzyme BpiI/Eco31I (BsaI isoenzyme $)$ & 2 \\
$\mathrm{ddH}_{2} \mathrm{O}$ & Up to 40 \\
\hline
\end{tabular}


Table S18 The ligation reaction system for HC-AcV-POT-BC1-BC2-UAS-YPettPGK1 library

\begin{tabular}{cc}
\hline Reagent & Volume $(\mu \mathrm{L})$ \\
\hline HC-AcV-POTB fragment with BpiI digestion & $100 \mathrm{ng}$ \\
UAS-YPet-tPGK1 fragment with Eco31I digestion & $50 \mathrm{ng}$ \\
$10 \times \mathrm{T} 4$ DNA Ligase Buffer & 5.0 \\
T4 DNA Ligase $(5 \mathrm{U} / \mu \mathrm{L})($ Thermo Scientific, EL0011) & 1.0 \\
$25 \mathrm{mM} \mathrm{ATP}$ & 1.0 \\
$\mathrm{ddH}_{2} \mathrm{O}$ & Up to 50 \\
\hline
\end{tabular}


Table S19 The digestion reaction system for HC-AcV-POT-BC1-BC2-UAS-YPettPGK1 library

\begin{tabular}{cc}
\hline Reagent & Volume $(\mu \mathrm{L})$ \\
\hline $10 \times$ Fast Digest & 4 \\
$20 \mathrm{mM}$ DTT & 2 \\
HC-AcV-POT-BC1-BC2-UAS-YPet-tPGK1 library & $2 \mu \mathrm{g}$ \\
Esp3I (BsmBI, Thermo Scientific, FD0454) & 2 \\
$\mathrm{ddH}_{2} \mathrm{O}$ & Up to 40 \\
\hline
\end{tabular}


Table S20 Number of cells sorted into each bin in FapR-fapO based malonyl-CoA biosensor library sorting experiment

\begin{tabular}{ccccccccc}
\hline Sample & P2 & P3 & P4 & P5 & P6 & P7 & P8 & P9 \\
\hline Lib_0A\&0 & 10000 & 40000 & 50000 & 50000 & 50000 & 50000 & 50000 & 50000 \\
B & 0 & 0 & 0 & 0 & 0 & 0 & 0 & 0 \\
Lib_1A\&1 & 20000 & 40000 & 50000 & 50000 & 50000 & 50000 & 50000 & 50000 \\
B & 0 & 0 & 0 & 0 & 0 & 0 & 0 & 0 \\
Lib_2A\&2 & 20000 & 40000 & 50000 & 50000 & 50000 & 50000 & 50000 & 50000 \\
B & 0 & 0 & 0 & 0 & 0 & 0 & 0 & 0 \\
Lib_3A\&3 & 20000 & 40000 & 50000 & 50000 & 50000 & 50000 & 50000 & 50000 \\
B & 0 & 0 & 0 & 0 & 0 & 0 & 0 & 0 \\
Lib_5A\&5 & 30000 & 40000 & 50000 & 50000 & 50000 & 50000 & 50000 & 50000 \\
B & 0 & 0 & 0 & 0 & 0 & 0 & 0 & 0 \\
Lib_8A\&8 & 30000 & 40000 & 50000 & 50000 & 50000 & 50000 & 50000 & 50000 \\
B & 0 & 0 & 0 & 0 & 0 & 0 & 0 & 0 \\
\hline
\end{tabular}


Table S21 Data quality of each NGS library ${ }^{\#}$

\begin{tabular}{ccccccc}
\hline Sample & $\begin{array}{c}\text { Raw read } \\
\text { count }\end{array}$ & $\begin{array}{c}\text { Yield } \\
\text { (Gbases) }\end{array}$ & $\begin{array}{c}\text { Q30 } \\
(\%)\end{array}$ & $\begin{array}{c}\text { Remain } \\
\text { ed ratio }\end{array}$ & $\begin{array}{c}\text { Mapped } \\
\text { read count }\end{array}$ & Note \\
\hline FapR-Lib0A & $31,446,612$ & 9.434 & 94.61 & 0.844 & 26555200 & Sequence 1 \\
FapR-Lib0B & $32,565,692$ & 9.7697 & 91.93 & 0.656 & 21360628 & Sequence 1 \\
FapR-Lib1A & $33,541,416$ & 10.0624 & 94.06 & 0.808 & 27113992 & Sequence 1 \\
FapR-Lib1B & $32,681,659$ & 9.8045 & 93.77 & 0.829 & 27097539 & Sequence 1 \\
FapR-Lib2A & $30,205,278$ & 9.0616 & 94.37 & 0.834 & 25203969 & Sequence 1 \\
FapR-Lib2B & $28,739,496$ & 8.6218 & 93.26 & 0.803 & 23067655 & Sequence 1 \\
FapR-Lib3A & $29,883,846$ & 8.9652 & 93.19 & 0.789 & 23563601 & Sequence 1 \\
FapR-Lib3B & $29,234,103$ & 8.7702 & 93.59 & 0.832 & 24328837 & Sequence 1 \\
FapR-Lib5A & $29,607,124$ & 8.8821 & 93.62 & 0.803 & 23783320 & Sequence 1 \\
FapR-Lib5B & $31,106,960$ & 9.3321 & 93.55 & 0.822 & 25580350 & Sequence 1 \\
FapR-Lib8A & $21,492,038$ & 6.4476 & 93.81 & 0.804 & 17289776 & Sequence 1 \\
FapR-Lib8B & $27,711,470$ & 8.3134 & 93.53 & 0.826 & 22886273 & Sequence 1 \\
FapR-Lib0A & $26,252,730$ & 7.8758 & 94.2 & 1.012 & 26555200 & Sequence 2 \\
FapR-Lib0B & $39,109,538$ & 11.7329 & 91.7 & 0.564 & 22050253 & Sequence 2 \\
FapR-Lib1A & $37,477,477$ & 11.2432 & 93.65 & 0.684 & 25627993 & Sequence 2 \\
FapR-Lib1B & $41,620,531$ & 12.4862 & 93.99 & 0.724 & 30120509 & Sequence 2 \\
FapR-Lib2A & $28,313,465$ & 8.494 & 93.93 & 1.225 & 34680313 & Sequence 2 \\
FapR-Lib2B & $27,198,936$ & 8.1597 & 93.49 & 0.864 & 23499509 & Sequence 2 \\
FapR-Lib3A & $29,675,431$ & 8.9026 & 93.52 & 0.737 & 21877094 & Sequence 2 \\
FapR-Lib3B & $28,030,485$ & 8.4091 & 93.81 & 0.851 & 23851472 & Sequence 2 \\
FapR-Lib5A & $27,633,681$ & 8.2901 & 93.69 & 0.846 & 23364685 & Sequence 2 \\
FapR-Lib5B & $27,868,233$ & 8.3605 & 93.78 & 0.798 & 22235790 & Sequence 2 \\
FapR-Lib8A & $27,677,638$ & 8.3033 & 93.6 & 0.830 & 22968046 & Sequence 2 \\
FapR-Lib8B & $30,320,520$ & 9.0962 & 93.67 & 0.735 & 22271260 & Sequence 2 \\
\hline & & & & & & \\
\hline
\end{tabular}

\#For the exact definition of each bin, see BioProject PRJNA782357 at NCBI SRA site.

\& Merging of paired-end reads, trimming low quality nucleotides from two ends,

eliminating reads with overall low quality (see Methods for details)

${ }^{*}$ Mapping to the in silico library (5,184 possible designs) (see Methods for details) 
Table S22 Information about Gate Barcodes

\begin{tabular}{|c|c|c|c|c|}
\hline Bin & Primer A & Gate Barcode & Primer B & Gate Barcode \\
\hline $\mathrm{P} 2$ & NGS_01 & CAAAATAC & NGS_02 & TCACTGC \\
\hline P3 & NGS_03 & CAACCGC & NGS_04 & TCAGAGA \\
\hline $\mathrm{P} 4$ & NGS_05 & CAAGTCT & NGS_06 & TCCAAGC \\
\hline P5 & NGS_07 & CAATGGA & NGS_08 & TCCCCGA \\
\hline P6 & NGS_09 & CACAATT & NGS_10 & TCCGCAG \\
\hline $\mathrm{P} 7$ & NGS_11 & CA $\underline{\mathrm{CAGAG}}$ & NGS_12 & TCCTTTA \\
\hline P8 & NGS_13 & CACCAAA & NGS_14 & TCGATTG \\
\hline P9 & NGS_15 & CACTCGG & NGS_16 & TCGCGAT \\
\hline
\end{tabular}


Table S23 The median value of the fluorescence intensity $I_{j}$ of each bin in FapR-fapO based malonyl-CoA biosensor library sorting experiment

\begin{tabular}{ccccccccc}
\hline Gate & P2 & P3 & P4 & P5 & P6 & P7 & P8 & P9 \\
\hline Lib_0A & 0.79967 & 0.51731 & 0.20461 & - & - & - & - & - \\
& & & & 0.10878 & 0.42263 & 0.73458 & 1.04987 & 1.49658 \\
Lib_0B & 0.80362 & 0.51834 & 0.2059 & - & - & - & - & - \\
& & & & 0.10788 & 0.42136 & 0.73339 & 1.04931 & 1.48922 \\
Lib_1A & 0.81916 & 0.51899 & 0.20433 & - & - & -0.7342 & - & - \\
Lib_1B & 0.81585 & 0.51865 & 0.20441 & - & - & - & - & - \\
& & & & 0.10954 & 0.42235 & 0.73364 & 1.04993 & 1.50406 \\
Lib_2A & 0.82155 & 0.51839 & 0.20336 & - & - & - & - & - \\
& & & & 0.10981 & 0.42189 & 0.73357 & 1.05061 & 1.52892 \\
Lib_2B & 0.81977 & 0.51883 & 0.20352 & - & - & - & - & -1.5378 \\
Lib_3A & 0.82207 & 0.51898 & 0.20381 & - & - & - & - & - \\
& & & & 0.10915 & 0.42131 & 0.73358 & 1.05026 & 1.53421 \\
Lib_3B & 0.82228 & 0.5187 & 0.20315 & -0.1097 & - & - & - & -1.5369 \\
& & & & & 0.42164 & 0.73378 & 1.05097 & \\
Lib_5A & 0.81668 & 0.51597 & 0.2005 & -0.1116 & - & -0.7359 & - & -1.5523 \\
Lib_5B & 0.81592 & 0.51621 & 0.20077 & - & - & - & - & - \\
Lib_8A & 0.81485 & 0.51502 & 0.19997 & - & -0.4232 & - & - & - \\
Lib_8B & 0.81559 & 0.51668 & 0.20093 & - & - & - & -1.0531 & -1.5477 \\
\hline & & & & 0.11127 & 0.42261 & 0.73569 & & \\
\hline
\end{tabular}


Table S24 The ratio of cells for each bin in FapR-fapO based malonyl-CoA biosensor library sorting experiment

\begin{tabular}{ccccccccc}
\hline Sample & P2 & P3 & P4 & P5 & P6 & P7 & P8 & P9 \\
\hline Lib_0A & 0.0213 & 0.0529 & 0.1067 & 0.1130 & 0.1008 & 0.0898 & 0.2150 & 0.3001 \\
& 7 & 5 & 6 & 0 & 3 & 7 & 6 & 7 \\
Lib_0B & 0.0218 & 0.0547 & 0.1092 & 0.1126 & 0.0986 & 0.0888 & 0.2300 & 0.2839 \\
& 3 & 1 & 4 & 7 & 6 & 6 & 9 & 2 \\
Lib_1A & 0.0296 & 0.0743 & 0.1128 & 0.1175 & 0.0935 & 0.1129 & 0.2169 & 0.2422 \\
& 8 & 8 & 0 & 0 & 0 & 7 & 3 & 4 \\
Lib_1B & 0.0261 & 0.0700 & 0.1111 & 0.1146 & 0.0911 & 0.1064 & 0.2232 & 0.2573 \\
& 0 & 1 & 1 & 6 & 4 & 3 & 2 & 4 \\
Lib_2A & 0.0361 & 0.0808 & 0.1115 & 0.1148 & 0.0956 & 0.1353 & 0.2094 & 0.2160 \\
& 2 & 8 & 9 & 6 & 9 & 3 & 8 & 3 \\
Lib_2B & 0.0332 & 0.0796 & 0.1113 & 0.1129 & 0.0943 & 0.1297 & 0.2105 & 0.2282 \\
& 1 & 0 & 0 & 1 & 5 & 9 & 9 & 4 \\
Lib_3A & 0.0389 & 0.0819 & 0.1141 & 0.1164 & 0.1013 & 0.1425 & 0.2011 & 0.2035 \\
& 8 & 3 & 1 & 2 & 0 & 6 & 1 & 9 \\
Lib_3B & 0.0373 & 0.0833 & 0.1099 & 0.1098 & 0.0980 & 0.1477 & 0.2075 & 0.2061 \\
& 5 & 7 & 0 & 6 & 6 & 3 & 8 & 5 \\
Lib_5A & 0.0452 & 0.0833 & 0.1114 & 0.1112 & 0.1155 & 0.1677 & 0.1875 & 0.1779 \\
& 4 & 4 & 4 & 2 & 7 & 0 & 7 & 2 \\
Lib_5B & 0.0386 & 0.0826 & 0.1087 & 0.1083 & 0.1081 & 0.1639 & 0.1979 & 0.1915 \\
& 6 & 5 & 4 & 8 & 2 & 9 & 6 & 0 \\
Lib_8A & 0.0461 & 0.0829 & 0.1097 & 0.1108 & 0.1190 & 0.1702 & 0.1842 & 0.1767 \\
& 1 & 9 & 8 & 3 & 6 & 7 & 6 & 1 \\
Lib_8B & 0.0401 & 0.0826 & 0.1080 & 0.1073 & 0.1118 & 0.1668 & 0.1959 & 0.1871 \\
& 8 & 3 & 8 & 3 & 8 & 1 & 7 & 1 \\
\hline
\end{tabular}




\section{Statistical information and software used in this work}

Plots were generated in Python 3 using the matplotlib (3.0.3) plotting libraries. All statistical analyses, data fitting, interpolation calculations and machine learning were performed using the scipy (1.3.1), numpy (1.16.5), pandas (0.24.2), sys (3.7.4) and sklearn (0.21.2) Python packages. 


\section{Supplementary References}

(1) Guo, Y., Dong, J., Zhou, T., Auxillos, J., Li, T., Zhang, W., Wang, L., Shen, Y., Luo, Y., Zheng, Y., Lin, J., Chen, G. Q., Wu, Q., Cai, Y., and Dai, J. (2015)

YeastFab: The design and construction of standard biological parts for metabolic engineering in Saccharomyces cerevisiae. Nucleic Acids Research 43, e88. 